\title{
STEKLOV TYPE PROBLEM IN A HALF-CYLINDER WITH A SMALL CAVITY
}

\author{
D.B. DAVLETOV, D.V. KOZHEVNIKOV
}

\begin{abstract}
In the work we consider a Steklov type problem for the Laplace operator in a $n$-dimensional cylinder with a small cavity. On the lateral surfaces one of three classic boundary conditions is imposed, the boundary of the cavity is subject to the Dirichlet condition, while on the base of the cylinder we impose the spectral Steklov condition. We prove the convergence theorems for the eigenvalues of this problems as the small parameter, the diameter of the cavity, tends to zero. We construct and justify the complete asymptotic expansions in the small parameter converging both to a simple or a double eigenvalue of the limiting problem, which is the problem without the cavity.
\end{abstract}

Keywords: half-cylinder, Steklov problem, eigenvalue, singular perturbation, small cavity, convergence, asymptotics.

Mathematics Subject Classification: 35J05, 35J25, 47A10, 47A55, 47A75, 47F05

\section{INTRODUCTION}

The studying of the eigenvalues of the boundary value problems for elliptic operators in domains with a small cavity has a rather long history. In [1], the estimate for the rate of the convergence of a Dirichlet eigenvalue for the Laplace operator in a three-dimensional domain with a small cavity. Later similar results were obtained in [2, 3, 4]. Then in [5], there were constructed complete asymptotic expansions for the first eigenvalues and corresponding eigenfunctions in two- and three-dimensional domains with small cavities.

The asymptotics of the solution to an elliptic boundary value problem with a small cavity on the spectrum of the limiting problem was obtained in [6]. The boundary value problems for elliptic operators in the elasticity theory with small holes were studied in works [7, 8, 9, 10]. In the case of Neumann conditions on the boundary of the small cavity, in [7], the complete asymptotic expansions for the eigenvalues of the perturbed boundary value problem were constructed. In work [8], there was proven the convergence of the eigenelements of the Dirichlet problem to the eigenelements of the corresponding limiting eigenvalue, while in [9, 10] there were constructed two-terms asymptotics w.r.t. a small parameter in two- and threedimensional cases, respectively. The complete asymptotics for the eigenvalues of the Steklov problem for the Laplace operator in domain with a small cavity were constructed in [11].

In the present work we study a Steklov type problem for the Laplace operator in an $n$ dimensional cylinder containing a small cavity. On the later surface we impose one of the classical boundary conditions (Dirichlet or Neumann or Robin condition), on the boundary of the small cavity we impose the Dirichlet condition, while the base of the half-cylinder is subject to the Steklov condition. Such issues arise in boundary value problems for the Laplace operator in a domain perforated along a part of the boundary [12]. Similar problems in half-strips and

D.B. Davletov, D.V. Kozhevnikov, The Problem of Steklov type in a half-Cylinder with a SMALL CAVITY.

(C) Davletov D.B., Kozhevnikov D.V. 2016.

The work is supported by RFBR (projects no. 16-31-00066-mol_a, 14-01-97024-r_povolzhe_a) .

Submitted June 9, 2016. 
half-cylinders with singularly perturbed boundary conditions arose earlier in problems with frequent alternation of boundary conditions [13, 14, 15].

In conclusion we mention that the emergence of the eigenvalues from the thresholds of the essential spectrum for the cylinders with small cavities and with the Dirichlet condition on the boundary of these small cavities was studied in [16, 17].

\section{Formulation OF THE MAin STATEMENTS}

Let $3 \leqslant n \in \mathbb{N}, \Sigma$ be an $(n-1)$-dimensional bounded domain with a smooth boundary, $\Pi:=\Sigma \times(a,+\infty),-\infty<a<0, \Sigma_{a}:=\Sigma \times\{a\},\{0\} \in \Pi, \omega$ be a bounded connected domain in $\mathbb{R}^{n}$ with a smooth boundary, $\omega_{\varepsilon}=\left\{x: \varepsilon^{-1} x \in \omega\right\}, 0<\varepsilon \ll 1, \Pi_{\varepsilon}=\Pi \backslash \overline{\omega_{\varepsilon}}$. We consider a singular perturbation of the following Steklov eigenvalue problem:

$$
\begin{aligned}
-\Delta \psi_{0} & =0, & & x \in \Pi, \quad \mathfrak{l} \psi_{0}:=\left(H \frac{\partial}{\partial \nu}+h\right) \psi_{0}=0, \quad x \in \partial \Pi \backslash \bar{\Sigma}_{a}, \\
\frac{\partial \psi_{0}}{\partial \nu} & =\lambda_{0} \psi_{0}, \quad x \in \Sigma_{a}, & &
\end{aligned}
$$

where $\nu$ is the outward normal, $H, h \geqslant 0, H+h \neq 0$. The perturbation is made by cutting out the small hole $\omega_{\varepsilon}$ in the half-cylinder and by imposing the Dirichlet condition on its boundary:

$$
\begin{aligned}
& -\Delta \psi_{\varepsilon}=0, \quad x \in \Pi_{\varepsilon}, \quad \mathfrak{l} \psi_{\varepsilon}=0, \quad x \in \partial \Pi \backslash \bar{\Sigma}_{a}, \\
& \frac{\partial \psi_{\varepsilon}}{\partial \nu}=\lambda_{\varepsilon} \psi_{\varepsilon}, \quad x \in \Sigma_{a}, \quad \psi_{\varepsilon}=0, \quad x \in \partial \omega_{\varepsilon} .
\end{aligned}
$$

The eigenfunctions are considered in the class of functions with a finite Dirichlet integral:

$$
\int_{\Pi}\left|\nabla \psi_{0}\right|^{2} d x<\infty, \quad \int_{\Pi_{\varepsilon}}\left|\nabla \psi_{\varepsilon}\right|^{2} d x<\infty .
$$

By the Fourier method one can show easily that the eigenvalues $\lambda_{0,1}<\lambda_{0,2} \leqslant \cdots \leqslant \lambda_{0, k} \leqslant \cdots$ and the associated orthonormalized in $L_{2}\left(\Sigma_{a}\right)$ eigenfunctions $\psi_{0, k}$ to Steklov problem (2.1) are determined by the identities:

$$
\lambda_{0, k}=\sqrt{\zeta_{k}}, \quad \psi_{0, k}(x)=\phi_{k}\left(x^{\prime}\right) \mathrm{e}^{-\sqrt{\zeta_{k}}\left(x_{n}-a\right)},
$$

where $x^{\prime}:=\left(x_{1}, \ldots, x_{n-1}\right), \zeta_{k}$ and $\phi_{k}$ are the eigenvalues and the associated normalized in $L_{2}(\Sigma)$ eigenfunctions to the boundary value problem:

$$
-\sum_{i=1}^{n-1} \frac{\partial^{2} \phi_{k}}{\partial x_{i}^{2}}=\zeta_{k} \phi_{k} \quad \text { in } \Sigma, \quad \mathfrak{l} \phi_{k}=0 \quad \text { on } \partial \Sigma .
$$

In the next section we shall prove

Theorem 2.1. Assume that the segment $\left[\lambda_{-}, \lambda_{+}\right]$contains no eigenvalues of Steklov problem 2.1). Then for all sufficiently small $\varepsilon$, this segment contains no eigenvalues of Steklov problem (2.2).

Assume the multiplicity of an eigenvalue $\lambda_{0}$ of Steklov problem (2.1) is equal to $d$. Then Steklov problem (2.2) has exactly $d$ eigenvalues $\lambda_{\varepsilon}^{(l)}, l=\overline{1, d}$, (counting the multiplicities) converging to $\lambda_{0}$ as $\varepsilon \rightarrow 0$.

The corresponding projectors $\mathcal{P}_{0}$ and $\mathcal{P}_{\varepsilon}$ satisfies the convergence $\mathcal{P}_{\varepsilon} \rightarrow \mathcal{P}_{0}$ as $\varepsilon \rightarrow 0$ in $L_{2}\left(\Sigma_{a}\right)$.

The main content of the work is the proof of the following Theorems 2.2 and 2.3 by the method of matching of asymptotic expansions [18, 19, 20].

Before we proceed to the formulations of these statements, we introduce some notations. Hereafter, $r=|x|,\left|S_{n}\right|$ is the area of the unit sphere in $\mathbb{R}^{n}$. 
By $z_{q}(x), q=\overline{0, n}$, we denote harmonic in $\mathbb{R}^{n} \backslash \bar{\omega}$ functions decaying at infinity and satisfying the boundary conditions:

$$
z_{0}(x)=1, \quad z_{m}(x)=x_{m}, \quad m=\overline{1, n} \quad \text { on } \quad \partial \omega .
$$

It is well-known that these functions have differentiable asymptotic expansions:

$$
z_{q}(x)=c_{q, 0} r^{-n+2}+\sum_{p=1}^{n} c_{q, p} x_{p} r^{-n}+\sum_{i=2}^{\infty} Z_{i}^{(q)}(x) r^{-2 i-n+2}, \quad r \rightarrow \infty,
$$

where $Z_{i}^{(q)}(x)$ are homogeneous harmonic polynomials of degree $i$ with an index $q$. The constant $c_{0,0}=c(\omega)>0$ is called the harmonic capacity, while the constants $c_{m, q}, m, q=\overline{1, n}$, are called the coefficients of the dipole form associated with a polarization [21].

Integrating by parts in the right hand sides of the identities

$$
\begin{aligned}
& 0=\int_{\{r<R\} \backslash \omega}\left(x_{m}-z_{m}(x)\right) \Delta\left(x_{j}-z_{j}(x)\right) \mathrm{d} x, \quad j, m=\overline{1, n}, \\
& 0=\int_{\{r<R\} \backslash \omega}\left(x_{m}-z_{m}(x)\right) \Delta\left(1-z_{0}(x)\right) \mathrm{d} x, \quad m=\overline{1, n},
\end{aligned}
$$

as $R \rightarrow+\infty$, it is easy to show that

$$
c_{m, j}=c_{j, m}, \quad j, m=\overline{1, n}, \quad(n-2) c_{m, 0}=c_{0, m}, \quad m=\overline{1, n} .
$$

By these identities, the $n \times n$-matrices $C(\omega)$ and $\widetilde{C}(\omega)$ with the entries $c_{m, q}$ and

$$
\widetilde{c}_{m, q}=c_{m, q}-\frac{c_{m, 0} c_{0, q}}{c(\omega)}, \quad m, q=\overline{1, n},
$$

are symmetric.

Theorem 2.2. Let $\lambda_{0}$ be a simple eigenvalue of Steklov problem (2.1), $\psi_{0}$ be the associated normalized in $L_{2}\left(\Sigma_{a}\right)$ eigenfunction.

Then the eigenvalue $\lambda_{\varepsilon}$ of perturbed Steklov problem (2.2) converging to $\lambda_{0}$ has the asymptotic expansion

$$
\lambda_{\varepsilon}=\lambda_{0}+\varepsilon^{n-2} \sum_{i=0}^{\infty} \varepsilon^{i} \lambda_{n-2+i}
$$

where

$$
\lambda_{n-2}=c(\omega)\left|S_{n}\right|(n-2) \psi_{0}^{2}(0)
$$

If $\psi_{0}(0)=0$, then

$$
\begin{aligned}
\lambda_{n-2} & =0, \\
\lambda_{n-1} & =0, \\
\lambda_{n} & =\left|S_{n}\right| \nabla \psi_{0}(0) C(\omega) \nabla \psi_{0}(0) .
\end{aligned}
$$

Remark 2.1. It is obvious that if $\omega$ is the unit ball centered at the origin, then

$$
z_{0}(x)=r^{-n+2}, \quad z_{m}(x)=x_{m} r^{-n}, \quad m=\overline{1, n} .
$$

By shifting and dilating the coordinate system, one can show easily that in the case when $\omega$ is a ball of radius $R$ centered at the point $(0, \ldots, 0, t)$, then matrices $C(\omega)$ and $\widetilde{C}(\omega)$ are diagonal and

$$
\begin{aligned}
c_{0,0} & =c(\omega)=R^{n-2}, \\
c_{n, n} & =R^{n-2}\left(R^{2}+(n-2) t^{2}\right), \quad c_{j, j}=\widetilde{c}_{j, j}=\widetilde{c}_{n, n}=R^{n}, \quad j=\overline{1, n-1 .}
\end{aligned}
$$


Therefore, in this the identities (2.8) and (2.11) become

$$
\begin{aligned}
\lambda_{n-2} & =R^{n-2}\left|S_{n}\right|(n-2) \psi_{0}^{2}(0), \\
\lambda_{n} & =R^{n-2}\left|S_{n}\right|\left(R^{2}\left|\nabla \psi_{0}(0)\right|^{2}+(n-2) t^{2}\left|\frac{\partial \psi_{0}}{\partial x_{n}}(0)\right|^{2}\right),
\end{aligned}
$$

respectively.

Remark 2.2. If $\zeta_{k}$ is a simple eigenvalues of boundary value problem (2.4), then by (2.3) identities (2.8) and (2.13) become

$$
\begin{aligned}
\lambda_{n-2} & =c(\omega)\left|S_{n}\right|(n-2) \mathrm{e}^{2 a \sqrt{\zeta_{k}}} \phi_{k}^{2}(0), \\
\lambda_{n-2} & =R^{n-2}\left|S_{n}\right|(n-2) \mathrm{e}^{2 a \sqrt{\zeta_{k}}} \phi_{k}^{2}(0), \\
\lambda_{n} & =R^{n-2}\left|S_{n}\right| \mathrm{e}^{2 a \sqrt{\zeta_{k}}}\left(R^{2}\left|\nabla^{\prime} \phi_{k}(0)\right|^{2}+\left(R^{2}+(n-2) t^{2}\right) \zeta_{k} \phi_{k}^{2}(0)\right),
\end{aligned}
$$

respectively, where $\nabla^{\prime} \phi$ stands for the vector with the components

$$
\frac{\partial \phi}{\partial x_{j}}, \quad j=\overline{1, n-1} .
$$

In the work we also construct the complete asymptotic expansion for the eigenfunction $\psi_{\varepsilon}$ of Steklov problem (2.2) associated with the eigenvalue $\lambda_{\varepsilon}$. However, by Theorem 2.1, the limiting value for $\psi_{\varepsilon}$ is known and it is unique up to the sign and is equal to the eigenfunction $\psi_{0}$ of the limiting Steklov problem (2.1).

Remark 2.3. In the work we consider both the case of simple and multiple eigenvalue. Since all the arguments for the double eigenvalue are easily adapted for the case of a n-multiple eigenvalue, for the simplicity of the presentation we construct the asymptotic expansions for a double eigenvalue.

If $\lambda_{0}$ is a double eigenvalue of problem (2.1), it follows from Theorem 2.1 that there are two options for the eigenvalues of perturbed problem $(2.2)$ converging to $\lambda_{0}$. These are either two simple eigenvalues or one double eigenvalue, or for different values of $\varepsilon$ one of these options hold true. And even if two simple eigenvalues $\lambda_{\varepsilon}^{(1)}$ and $\lambda_{\varepsilon}^{(2)}$ converge to $\lambda_{0}$, it does not imply that the associated normalized in $L_{2}\left(\Sigma_{a}\right)$ eigenfunctions $\psi_{\varepsilon}^{(1)}$ and $\psi_{\varepsilon}^{(2)}$ have limits. Theorem 2.1 just ensures that from each sequence $\varepsilon_{k} \rightarrow 0$ one can choose a subsequence $\varepsilon_{k_{m}} \rightarrow 0$ such that the convergence $\psi_{\varepsilon}^{(j)} \rightarrow \psi_{0}^{(j)}$ holds true in $L_{2}\left(\Sigma_{a}\right)$, where $\psi_{0}^{(j)}$ are orthonormalized in $L_{2}\left(\Sigma_{a}\right)$ eigenfunctions of problem (2.1) associated with $\lambda_{0}$. However, generally speaking, these limits can vary subject to the choice of sequence $\varepsilon_{k_{m}} \rightarrow 0$.

In the work we consider the most general case:

$$
\left|\psi_{0}^{(1)}(0)\right|+\left|\psi_{0}^{(2)}(0)\right| \neq 0
$$

Then it is obvious that these eigenvalues can be orthonormalized in $L_{2}\left(\Sigma_{a}\right)$ such that

$$
\psi_{0}^{(1)}(0) \neq 0, \quad \psi_{0}^{(2)}(0)=0 .
$$

We shall prove the following

Theorem 2.3. Let $\lambda_{0}$ be a double eigenvalue of problem (2.1), $\psi_{0}^{(1)}$ and $\psi_{0}^{(2)}$ be the associated eigenfunctions satisfying condition (2.14) and orthonormalized in $L_{2}\left(\Sigma_{a}\right)$ in accordance with (2.15).

Then there exist two simple eigenvalues $\lambda_{\varepsilon}^{(1)}$ and $\lambda_{\varepsilon}^{(2)}$ of perturbed Steklov problem (2.2) converging to $\lambda_{0}$. These eigenvalue have the asymptotic expansions

$$
\lambda_{\varepsilon}^{(1)}=\lambda_{0}+\varepsilon^{n-2} \sum_{i=0}^{\infty} \varepsilon^{i} \lambda_{n-2+i}^{(1)},
$$




$$
\lambda_{\varepsilon}^{(2)}=\lambda_{0}+\varepsilon^{n} \sum_{i=0}^{\infty} \varepsilon^{i} \lambda_{n+i}^{(2)},
$$

where

$$
\begin{aligned}
\lambda_{n-2}^{(1)} & =c(\omega)\left|S_{n}\right|(n-2)\left(\psi_{0}^{(1)}(0)\right)^{2}>0, \\
\lambda_{n}^{(2)} & =\left|S_{n}\right| \nabla \psi_{0}^{(2)}(0) \widetilde{C}(\omega) \nabla \psi_{0}^{(2)}(0) .
\end{aligned}
$$

The associated eigenfunctions $\psi_{\varepsilon}^{(j)}$ converge to $\psi_{0}^{(j)}$ in $L_{2}\left(\Sigma_{a}\right)$.

Remark 2.4. In particular, it follows from the above theorem that if condition (2.14) holds true, then under the considered perturbation, a double eigenvalue $\lambda_{0}$ splits into two simple eigenvalues and the associated eigenfunction converge to the eigenfunctions of Steklov problem (2.1) orthonormalized in $L_{2}\left(\Sigma_{a}\right)$ in accordance with (2.15).

Remark 2.5. If $\omega$ is a ball of radius $R$ centered at the point $(0, \ldots, 0, t)$, then by (2.12), identities (2.18) and (2.19) become

$$
\begin{gathered}
\lambda_{n-2}^{(1)}=R^{n-2}\left|S_{n}\right|(n-2)\left(\psi_{0}^{(1)}(0)\right)^{2}, \\
\lambda_{n}^{(2)}=R^{n}\left|S_{n}\right|\left|\nabla \psi_{0}^{(2)}(0)\right|^{2}>0,
\end{gathered}
$$

respectively.

Remark 2.6. If $\zeta_{k}=\zeta_{k+1}$ is a double eigenvalue of boundary value problem (2.4) and the associated eigenfunctions are orthonormalized in $L_{2}(\Sigma)$ such that $\phi_{k}\left(0^{\prime}\right)=0, \phi_{k+1}\left(0^{\prime}\right) \neq 0$, then by (2.3) identities (2.18) and (2.20) become

$$
\begin{aligned}
& \lambda_{n-2}^{(1)}=c(\omega)\left|S_{n}\right|(n-2) \mathrm{e}^{2 a \sqrt{\zeta_{k}}} \phi_{k}^{2}\left(0^{\prime}\right), \\
& \lambda_{n-2}^{(1)}=R^{n-2}\left|S_{n}\right|(n-2) \mathrm{e}^{2 a \sqrt{\zeta_{k}}} \phi_{k}^{2}\left(0^{\prime}\right), \\
& \lambda_{n}^{(2)}=R^{n}\left|S_{n}\right| \mathrm{e}^{2 a \sqrt{\zeta_{k}}}\left(\left|\nabla^{\prime} \phi_{k+1}\left(0^{\prime}\right)\right|^{2}+\zeta_{k} \phi_{k+1}^{2}\left(0^{\prime}\right)\right),
\end{aligned}
$$

respectively.

\section{Proof of Theorem 2.1}

We introduce the space $H^{1}(\Pi)$ as the completion by the norm

$$
\|w\|_{H^{1}(\Pi)}=\left(\int_{\Pi}|\nabla w|^{2} d x+\int_{\Sigma_{a}} w^{2} d x^{\prime}\right)^{1 / 2}
$$

of the functions in $C^{\infty}(\bar{\Pi})$ possessing a finite Dirichlet integral. The subset of functions in $H^{1}(\Pi)$ vanishing on $\partial \Pi \backslash \bar{\Sigma}_{a}$ is denoted by $H^{1}\left(\Pi ; \partial \Pi \backslash \bar{\Sigma}_{a}\right)$. The space $H^{1}\left(\Pi_{\varepsilon}\right)$ is defined as the completion by norm

$$
\|w\|_{H^{1}\left(\Pi_{\varepsilon}\right)}=\left(\int_{\Pi_{\varepsilon}}|\nabla w|^{2} d x+\int_{\Sigma_{a}} w^{2} d x^{\prime}\right)^{1 / 2}
$$

of the functions in $C^{\infty}\left(\bar{\Pi}_{\varepsilon}\right)$ possessing a finite Dirichlet integral. The subset of the functions in $H^{1}\left(\Pi_{\varepsilon}\right)$ vanishing on $\partial \omega_{\varepsilon}\left(\right.$ on $\left.\partial \omega_{\varepsilon} \cup \partial \Pi \backslash \bar{\Sigma}_{a}\right)$ is denoted by $H^{1}\left(\Pi_{\varepsilon} ; \partial \omega_{\varepsilon}\right)\left(\right.$ by $\left.H^{1}\left(\Pi_{\varepsilon} ; \partial \omega_{\varepsilon} \cup \partial \Pi \backslash \bar{\Sigma}_{a}\right)\right)$.

The boundary value problems

$$
-\Delta U_{0}=0, \quad x \in \Pi, \quad \mathfrak{l} U_{0}=0, \quad x \in \partial \Pi \backslash \bar{\Sigma}_{a}, \quad \frac{\partial U_{0}}{\partial \nu}+U_{0}=f, \quad x \in \Sigma_{a},
$$


and

$$
\begin{aligned}
& -\Delta U_{\varepsilon}=0, \quad x \in \Pi_{\varepsilon}, \quad \mathfrak{l} U_{\varepsilon}=0, \quad x \in \partial \Pi \backslash \bar{\Sigma}_{a}, \\
& \frac{\partial U_{\varepsilon}}{\partial \nu}+U_{\varepsilon}=f_{\varepsilon}, \quad x \in \Sigma_{a}, \quad U_{\varepsilon}=0, \quad x \in \partial \omega_{\varepsilon},
\end{aligned}
$$

will be treated in the generalized (weak) sense. That is, let $f, f_{\varepsilon} \in L_{2}\left(\Sigma_{a}\right)$. Then as $h=0$ (as $H=0$ ), an element of $H^{1}(\Pi)$ (an element of $H^{1}\left(\Pi ; \partial \Pi \backslash \bar{\Sigma}_{a}\right)$ ) is called a generalized solution to boundary value problem (3.3) if for each $v \in H^{1}(\Pi)$ (for each $v \in H^{1}\left(\Pi ; \partial \Pi \backslash \bar{\Sigma}_{a}\right)$ ) the following identity

$$
\int_{\Pi} \nabla U_{0} \nabla v d x+\int_{\Sigma_{a}} U_{0} v d x^{\prime}=\int_{\Sigma_{a}} f v d x^{\prime}
$$

holds true. As $h H \neq 0$, an element of $H^{1}(\Pi)$ is called a generalized solution to the boundary value problem 3.3 if for each $v \in H^{1}(\Pi)$ the identity

$$
\int_{\Pi} \nabla U_{0} \nabla v d x+H^{-1} h \int_{\partial \Pi \backslash \bar{\Sigma}_{a}} U_{0} v d s+\int_{\Sigma_{a}} U_{0} v d x^{\prime}=\int_{\Sigma_{a}} f v d x^{\prime}
$$

holds true. In the same way, as $h=0$ (as $H=0$ ), an element $U_{\varepsilon} \in H^{1}\left(\Pi_{\varepsilon} ; \partial \omega_{\varepsilon}\right.$ ) (an element $\left.U_{\varepsilon} \in H^{1}\left(\Pi_{\varepsilon} ; \partial \omega_{\varepsilon} \cup \partial \Pi \backslash \bar{\Sigma}_{a}\right)\right)$ is called a generalized solution to boundary value problem (3.4) if for each $v \in H^{1}\left(\Pi_{\varepsilon} ; \partial \omega_{\varepsilon}\right)$ (for each $v \in H^{1}\left(\Pi_{\varepsilon} ; \partial \omega_{\varepsilon} \cup \partial \Pi \backslash \bar{\Sigma}_{a}\right)$ ) the identity

$$
\int_{\Pi_{\varepsilon}} \nabla U_{\varepsilon} \nabla v d x+\int_{\Sigma_{a}} U_{\varepsilon} v d x^{\prime}=\int_{\Sigma_{a}} f_{\varepsilon} v d x^{\prime}
$$

holds. As $h H \neq 0$, an element $U_{\varepsilon} \in H^{1}\left(\Pi_{\varepsilon} ; \partial \omega_{\varepsilon}\right)$ is called a generalized solution to boundary value problem (3.4) if for each $v \in H^{1}\left(\Pi_{\varepsilon} ; \partial \omega_{\varepsilon}\right)$ the identity

$$
\int_{\Pi_{\varepsilon}} \nabla U_{\varepsilon} \nabla v d x+H^{-1} h \int_{\partial \Pi \backslash \bar{\Sigma}_{a}} U_{\varepsilon} v d s+\int_{\Sigma_{a}} U_{\varepsilon} v d x^{\prime}=\int_{\Sigma_{a}} f_{\varepsilon} v d x^{\prime}
$$

holds true.

It is obvious that if we extend a function in $H^{1}\left(\Pi_{\varepsilon} ; \partial \omega_{\varepsilon}\right)$ (in $H^{1}\left(\Pi_{\varepsilon} ; \partial \omega_{\varepsilon} \cup \partial \Pi \backslash \bar{\Sigma}_{a}\right)$ ) by zero in $\bar{\omega}_{\varepsilon}$, it belongs to $H^{1}(\Pi)$ (belongs $H^{1}\left(\Pi ; \partial \Pi \backslash \bar{\Sigma}_{a}\right)$ ). For these extensions, we keep their original notations.

Substituting $v=U_{0}$ and $v=U_{\varepsilon}$ into (3.5), (3.6) and into (3.7), (3.8), we obtain the apriori estimates

$$
\left\|U_{0}\right\|_{H^{1}(\Pi)} \leqslant\|f\|_{L_{2}(\Sigma)}, \quad\left\|U_{\varepsilon}\right\|_{H^{1}(\Pi)} \leqslant\left\|f_{\varepsilon}\right\|_{L_{2}(\Sigma)} .
$$

It implies the uniqueness of solutions to boundary value problems $(3.3)$ and $(3.4)$.

Separating variables, we show easily that the sought solution to boundary value problem (3.3) can be represented as

$$
U_{0}(x)=\sum_{j=1}^{\infty} \frac{\left(f, \phi_{j}\right)_{0}}{\zeta_{j}+1} \phi_{j}\left(x^{\prime}\right) \mathrm{e}^{-\sqrt{\zeta_{j}}\left(x_{n}-a\right)},
$$

where $(u, v)_{0}$ is the scalar product in $L_{2}(\Sigma)$.

Let us prove the solvability of boundary value problem (3.4). We denote by $(u, v)_{1}$ the scalar product in $H^{1}\left(\Pi_{\varepsilon}\right)$. Then integral identity (3.7) is written as

$$
\left(U_{\varepsilon}, v\right)_{1}=\int_{\Sigma_{a}} f_{\varepsilon} v d x^{\prime}
$$

For each fixed $f_{\varepsilon} \in L_{2}(\Sigma)$, the right hand side is a linear bounded functional on the Hilbert space $H^{1}\left(\Pi_{\varepsilon} ; \partial \omega_{\varepsilon}\right)$ (on the Hilbert space $H^{1}\left(\Pi_{\varepsilon} ; \partial \omega_{\varepsilon} \cup \partial \Pi \backslash \bar{\Sigma}_{a}\right)$ ). This is by the Riesz theorem 
there exists the unique element $F_{\varepsilon} \in H^{1}\left(\Pi_{\varepsilon} ; \partial \omega_{\varepsilon}\right)$ (element of $F_{\varepsilon} \in H^{1}\left(\Pi_{\varepsilon} ; \partial \omega_{\varepsilon} \cup \partial \Pi \backslash \bar{\Sigma}_{a}\right)$ ) such that

$$
\int_{\Sigma_{a}} f_{\varepsilon} v d x^{\prime}=\left(F_{\varepsilon}, v\right)_{1}
$$

for each $v \in H^{1}\left(\Pi_{\varepsilon} ; \partial \omega_{\varepsilon}\right)$ (each $\left.v \in H^{1}\left(\Pi_{\varepsilon} ; \partial \omega_{\varepsilon} \cup \partial \Pi \backslash \bar{\Sigma}_{a}\right)\right)$. By 3.11$)$ it follows that $U_{\varepsilon}=F_{\varepsilon}$, that is, boundary value problem $(3.4)$ is uniquely solvable as $h=0$ and $H=0$. In the same way, by using integral identity (3.8) we prove the unique solvability of boundary value problem (3.4) as $h H \neq 0$.

We denote by $T_{0}: L_{2}\left(\Sigma_{a}\right) \rightarrow L_{2}\left(\Sigma_{a}\right)$ the linear operator mapping a function $f$ into the restriction of solution $U_{0}$ of boundary value problem $(3.3)$ on $\Sigma_{a}$, that is, (see (3.10) )

$$
T_{0} f:=\sum_{j=1}^{\infty} \frac{\left(f, \phi_{j}\right)_{0}}{\zeta_{j}+1} \phi_{j}\left(x^{\prime}\right) .
$$

And by $T_{\varepsilon}: L_{2}\left(\Sigma_{a}\right) \rightarrow L_{2}\left(\Sigma_{a}\right)$ we denote the linear operator mapping a function $f_{\varepsilon}$ into the restriction of solution $U_{\varepsilon}$ of boundary value problem (3.4) on $\Sigma_{a}$.

Since $f_{k} \rightarrow f$ in $L_{2}\left(\Sigma_{a}\right)$ as $k \rightarrow \infty$, and the operator $T_{0}$ is compact by the compactness of the embedding of $H^{1}(\Pi)$ in $L_{2}\left(\Sigma_{a}\right)$, we have the convergence

$$
T_{0} f_{k} \rightarrow T_{0} f \quad \text { in } \quad L_{2}\left(\Sigma_{a}\right) \text { as } k \rightarrow \infty \text {. }
$$

Lemma 3.1. Let $v$ be an arbitrary function in $C^{\infty}(\bar{\Pi})$ (in $C^{\infty}(\bar{\Pi})$ vanishing on $\left.\partial \Pi \backslash \bar{\Sigma}_{a}\right)$ possessing a finite Dirichlet integral. Then there exist functions $v_{\varepsilon} \in H^{1}\left(\Pi_{\varepsilon} ; \partial \omega_{\varepsilon}\right.$ ) (functions $\left.v_{\varepsilon} \in H^{1}\left(\Pi_{\varepsilon} ; \partial \omega_{\varepsilon} \cup \partial \Pi \backslash \bar{\Sigma}_{a}\right)\right)$ such that $\left\|v-v_{\varepsilon}\right\|_{H^{1}(\Pi)} \rightarrow 0$ as $\varepsilon \rightarrow 0$.

Proof. Without loss of generality, we assume that the domain $\omega_{\varepsilon}$ is located in the ball of radius $\varepsilon$ centered at the origin. Let $\tilde{\chi}(t)$ be an infinitely differentiable cut-off function vanishing as $t \leqslant 1$ and being one as $t \geqslant 2$. It is easy to check that the functions $v_{\varepsilon}(x)=\tilde{\chi}\left(\frac{|x|}{\varepsilon}\right) v(x)$ satisfy the statement of the lemma.

For $R>0$ we denote $\Pi(R)=\Sigma \times(a, R)$,

$$
\|w\|_{H^{1}(\Pi(R))}=\left(\int_{\Pi(R)}|\nabla w|^{2} d x+\int_{\Sigma_{a}} w^{2} d x^{\prime}\right)^{1 / 2} .
$$

Since, obviously, $\|w\|_{H^{1}(\Pi(R))} \leqslant\|w\|_{H^{1}(\Pi)}$, and $\|w\|_{W_{2}^{1}(\Pi(R))} \leqslant C(R)\|w\|_{H^{1}(\Pi(R))}$ by [22, Ch. III, Sect. 5, Thm. 5], then

$$
\|w\|_{W_{2}^{1}(\Pi(R))} \leqslant C(R)\|w\|_{H^{1}(\Pi)} .
$$

Lemma 3.2. If

$$
f_{\varepsilon} \rightarrow f \text { in } L_{2}\left(\Sigma_{a}\right) \text { as } \varepsilon \rightarrow 0,
$$

for the solutions to boundary value problems (3.3) and (3.4) the convergence

$$
T_{\varepsilon} f_{\varepsilon} \rightarrow T_{0} f \quad \text { in } L_{2}\left(\Sigma_{a}\right) \text { as } \varepsilon \rightarrow 0 \text {, }
$$

holds true.

Proof. By the weak compactness of a bounded set in a Hilbert space (see, for instance, [23, Ch. 2, Sect. 3]), estimates (3.9) and (3.14) and the compact embedding of $W_{2}^{1}(\Pi(R))$ into $L_{2}\left(\Sigma_{a}\right)$, from each sequence $\varepsilon_{k} \underset{k \rightarrow \infty}{\rightarrow} 0$ we can choose a subsequence (which, without loss of generality, is assumed to coincide with sequence $\left.\left\{\varepsilon_{k}\right\}\right)$ such that on this sequence

$$
\begin{aligned}
& U_{\varepsilon} \rightarrow U_{*} \quad \text { in } \quad H^{1}(\Pi) \quad \text { as } \quad \varepsilon=\varepsilon_{k} \rightarrow 0, \\
& U_{\varepsilon} \rightarrow U_{*} \quad \text { in } \quad L_{2}\left(\Sigma_{a}\right) \quad \text { as } \quad \varepsilon \rightarrow 0,
\end{aligned}
$$


and $U_{*} \in H^{1}(\Pi)$ if $U_{\varepsilon} \in H^{1}\left(\Pi_{\varepsilon} ; \partial \omega_{\varepsilon}\right)$ and $U_{*} \in H^{1}\left(\Pi ; \partial \Pi \backslash \bar{\Sigma}_{a}\right)$ if $U_{\varepsilon} \in H^{1}\left(\Pi_{\varepsilon} ; \partial \omega_{\varepsilon} \cup \partial \Pi \backslash \bar{\Sigma}_{a}\right)$.

It remains to show that $U_{*}=U_{0}$. Then the arbitrary choice of the original sequence $\varepsilon_{k} \underset{k \rightarrow \infty}{\rightarrow} 0$ will imply convergence 3.15 . Let $v$ be an arbitrary function in $C^{\infty}(\bar{\Pi})$ (in $C^{\infty}(\bar{\Pi})$ vanishing on $\partial \Pi \backslash \bar{\Sigma}_{a}$ ) possessing a finite Dirichlet integral, the functions $v_{\varepsilon}$ satisfy Lemma 3.1. Passing to the limit as $\varepsilon \rightarrow 0$ in (3.7) and (3.8) for $v=v_{\varepsilon}$, by (3.16) and by Lemma 3.1, by the definition of the space $H^{1}(\Pi)$ and $H^{1}\left(\Pi ; \partial \Pi \backslash \bar{\Sigma}_{a}\right)$ we obtain that the function $U_{*}$ is a generalized solution to boundary value problem (3.3). And since the solution to boundary value problem (3.3) is unique, we have $U_{*}=U_{0}$.

Lemma 3.3. As $\varepsilon \rightarrow 0$, the convergence $T_{\varepsilon} \rightarrow T_{0}$ holds true in the sense of the operator norm.

Proof. In order to prove the lemma, it is sufficient to prove the uniform convergence

$$
\left\|T_{\varepsilon} f-T_{0} f\right\|_{L_{2}\left(\Sigma_{a}\right)} \underset{\varepsilon \rightarrow 0}{\rightrightarrows} 0
$$

for functions $f$ normalized in $L_{2}\left(\Sigma_{a}\right)$.

We assume the opposite. Then there exists a number $\delta>0$, a sequence $\varepsilon_{k} \rightarrow 0$ as $k \rightarrow \infty$ and a sequence of normalized in $L_{2}\left(\Sigma_{a}\right)$ functions $f_{k}$ such that

$$
\left\|T_{\varepsilon_{k}} f_{k}-T_{0} f_{k}\right\|_{L_{2}\left(\Sigma_{a}\right)}>\delta \text {. }
$$

Since a bounded set is weakly compact, without loss of generality we can assume that

$$
f_{k} \rightarrow f
$$

in $L_{2}\left(\Sigma_{a}\right)$. By (3.18) and the triangle inequality we get the inequality

$$
\left\|T_{\varepsilon_{k}} f_{k}-T_{0} f\right\|_{L_{2}\left(\Sigma_{a}\right)}+\left\|T_{0} f-T_{0} f_{k}\right\|_{L_{2}\left(\Sigma_{a}\right)}>\delta,
$$

which contradicts 3.13 and Lemma 3.2 .

Since boundary value problems $(3.3)$ and $(3.4)$ are uniquely solvable, there exist the inverse operators $S_{0}=T_{0}^{-1}$ and $S_{\varepsilon}=T_{\varepsilon}^{-1}$ defined in $L_{2}(\Sigma)$. This lemma and [23, Ch. 4, Sect. 2] imply the following statement.

Lemma 3.4. As $\varepsilon \rightarrow 0$, the operator $S_{\varepsilon}$ converges to the operator $S_{0}$ in the generalized sense.

Proof of Theorem 2.1. It follows from the definition of the operators $S_{0}$ and $S_{\varepsilon}$ that the eigenvalues $\Lambda_{0}$ and $\Lambda_{\varepsilon}$ of these operators and the eigenvalues $\lambda_{0}$ and $\lambda_{\varepsilon}$ of Steklov problems (2.1) and (2.2) are related by the identities $\lambda_{0}=\Lambda_{0}-1$ and $\lambda_{\varepsilon}=\Lambda_{\varepsilon}-1$, while the associated normalized in $L_{2}\left(\Sigma_{a}\right)$ eigenfunctions coincide. By Lemma 3.4 and [23, Ch. 4, Thm. 3.16] this implies the statement of the theorem.

\section{Auxiliary statements}

We recall that $X_{k}^{(q)}(x), Y_{k}^{(q)}(x)$ and $Z_{k}^{(q)}(x)$ are homogeneous harmonic polynomials of degree $k$ with an index $q$ indicating the function, for which they are written.

Lemma 4.1. For each harmonic polynomial $\widetilde{V}$ there exists a solution $V \in C^{\infty}\left(\mathbb{R}^{n} \backslash \bar{\omega}\right)$ to the boundary value problem

$$
\Delta V=0, \quad x \in \mathbb{R}^{n} \backslash \bar{\omega}, \quad V=0, \quad x \in \partial \omega,
$$

having a differentiable asymptotic expansion

$$
V(x)=\widetilde{V}(x)+\sum_{i=0}^{\infty} Z_{i}(x) r^{-2 i-n+2}, \quad r \rightarrow \infty .
$$


Proof. It follows from [18, Ch. 3, Sect. 1] that the boundary value problem

$$
\Delta v=0, \quad x \in \mathbb{R}^{n} \backslash \bar{\omega}, \quad v=-\widetilde{V}, \quad x \in \partial \omega,
$$

is solvable in the class of decaying as $r \rightarrow \infty$ function and the differentiable asymptotic expansion of the solution is of the form

$$
v(x)=\sum_{i=0}^{\infty} Z_{i}(x) r^{-2 i-n+2}, \quad r \rightarrow \infty .
$$

Therefore, Steklov problem 4.1 has the solution $V=\widetilde{V}+v$ with asymptotics 4.2 as $r \rightarrow$ $\infty$.

We denote by $\mathcal{A}$ the subset of functions $u(x)$ in the class $C^{\infty}(\bar{\Pi} \backslash\{0\})$ such that $u(x) \widetilde{\chi}(r R)$ is an element of $H^{1}(\Pi)$ for each sufficiently large $R>0$. We recall that $\tilde{\chi}(t)$ is an infinitely differentiable cut-off function vanishing as $t \leqslant 1$ and being equal to one as $t \geqslant 2$.

Lemma 4.2. Let $\lambda_{0}$ be a simple eigenvalue of Steklov problem (2.1), $Y_{j}(x)$ be an arbitrary harmonic polynomial, $F \in C^{\infty}\left(\Sigma_{a}\right)$. Then there exists a constant $\mu$, for which the Steklov problem

$$
\begin{aligned}
-\Delta E & =0, \quad x \in \Pi \backslash\{0\}, \quad \mathfrak{E}=0, \quad x \in \partial \Pi \backslash \bar{\Sigma}_{a}, \\
\frac{\partial E}{\partial \nu} & =\lambda_{0} E+F+\mu \psi_{0}, \quad x \in \Sigma_{a},
\end{aligned}
$$

is solvable and a solution is orthogonal to the function $\psi_{0}$ in $L_{2}\left(\Sigma_{a}\right)$, and $E \in \mathcal{A}$ and has the following differentiable asymptotic expansion

$$
E(x)=Y_{j}(x) r^{-2 j-n+2}+\sum_{k=0}^{\infty} X_{k}(x), \quad x \rightarrow 0 .
$$

Proof. We seek $E(x)$ as

$$
E(x)=(1-\tilde{\chi}(r R)) Y_{j}(x) r^{-2 j-n+2}+\widetilde{E}(x),
$$

where $R$ is a sufficiently large positive number. Substituting (4.5) into 4.3), we obtain the problem for $\widetilde{E}(x)$ :

$$
\begin{aligned}
-\Delta \widetilde{E} & =\widetilde{F}, \quad x \in \Pi \backslash\{0\}, \quad \mathfrak{l} \widetilde{E}=0, \quad x \in \partial \Pi \backslash \bar{\Sigma}_{a}, \\
\frac{\partial \widetilde{E}}{\partial \nu} & =\lambda_{0} \widetilde{E}+F+\mu \psi_{0}, \quad x \in \Sigma_{a},
\end{aligned}
$$

where $\widetilde{F} \in C_{0}^{\infty}(\Pi)$. Separating variables, it is easy to show the existence of a number $\mu$, for which a solution $\widetilde{E}(x)$ to problem 4.6 exists, belongs to $C^{\infty}(\bar{\Pi}) \cap H^{1}(\Pi)$ and is defined up to a term $\alpha \psi_{0}(x)$ with an arbitrary $\alpha$. Then for an appropriate choice of $\alpha$ function (4.5) satisfies the statement of the lemma.

It follows from the definition of spaces $H^{1}(\Pi)$ and $\mathcal{A}$ that for $\psi_{0}(x)$ and each function $E(x)$ solving problem 4.3, the identities

$$
\left|\psi_{0}(x) \frac{\partial E}{\partial x_{n}}(x)\right|+\left|E(x) \frac{\partial \psi_{0}}{\partial x_{n}}(x)\right| \underset{x_{n} \rightarrow \infty}{\longrightarrow} 0,
$$

hold true.

Corollary 1. There exist functions $E_{q} \in \mathcal{A}, q=\overline{0, n}$, as $r \rightarrow 0$, having the differentiable asymptotic expansions:

$$
E_{0}=r^{-n+2}+\sum_{k=0}^{\infty} X_{k}(x)
$$




$$
E_{m}=x_{m} r^{-n}+\sum_{k=0}^{\infty} X_{k}(x), \quad m=\overline{1, n},
$$

and being the solutions to the boundary value problems

$$
\begin{aligned}
-\Delta E_{q} & =0, \quad x \in \Pi \backslash\{0\}, \quad \mathfrak{l} E_{q}=0, \quad x \in \partial \Pi \backslash \bar{\Sigma}_{a}, \\
\frac{\partial E_{q}}{\partial \nu} & =\lambda_{0} E_{q}+\mu_{q} \psi_{0}, \quad x \in \Sigma_{a},
\end{aligned}
$$

as

$$
\begin{aligned}
\mu_{0} & =\left|S_{n}\right|(n-2) \psi_{0}(0), \\
\mu_{m} & =\left|S_{n}\right| \frac{\partial \psi_{0}}{\partial x_{m}}(0), \quad m=\overline{1, n} .
\end{aligned}
$$

Proof. In view of Lemma 4.2 it is sufficient to check identities (4.11) and (4.12). Let us prove (4.11). Let $B_{\delta}$ be the ball of radius $\delta \ll 1$ centered at the origin. Then integrating twice by parts, we obtain

$$
\begin{aligned}
0 & =\int_{\Pi\left(\delta^{-1}\right) \backslash B_{\delta}} \Delta E_{0} \psi_{0} d x= \\
& =\int_{x_{n}=\delta^{-1}}\left(\frac{\partial E_{0}}{\partial x_{n}} \psi_{0}-\frac{\partial \psi_{0}}{\partial x_{n}} E_{0}\right) d x^{\prime}-\int_{r=\delta}\left(\frac{\partial E_{0}}{\partial r} \psi_{0}-\frac{\partial \psi_{0}}{\partial r} E_{0}\right) d s+\mu_{0} .
\end{aligned}
$$

The Taylor series of the function $\psi_{0}(x)$ at zero is

$$
\begin{aligned}
\psi_{0}(x) & =\sum_{k=0}^{\infty} X_{k}^{(0)}(x), \quad r \rightarrow 0, \\
X_{0}^{(0)}(x) & =\psi_{0}(0), \quad X_{1}^{(0)}(x)=\sum_{m=1}^{n} \frac{\partial \psi_{0}}{\partial x_{m}}(0) x_{m} .
\end{aligned}
$$

Substituting (4.7), (4.8) and (4.14) into (4.13) and passing to the limit as $\delta \rightarrow 0$, we obtain identity 4.11. Identity 4.12 can be proved in the same way.

Similar to Lemma 4.2 we prove

Lemma 4.3. Let $\lambda_{0}$ be a double eigenvalue of Steklov problem (2.1), $\psi_{0}^{(1)}$ and $\psi_{0}^{(2)}$ be the associated orthonormalized in $L_{2}\left(\Sigma_{a}\right)$ eigenfunctions, $Y_{j}(x)$ be an arbitrary harmonic polynomial, $F \in C^{\infty}\left(\bar{\Sigma}_{a}\right)$. Then there exist constants $\mu^{(i)}$, for which the Steklov problem

$$
\begin{aligned}
-\Delta E & =0, \quad x \in \Pi \backslash\{0\}, \quad \mathfrak{l} E=0, \quad x \in \partial \Pi \backslash \bar{\Sigma}_{a}, \\
\frac{\partial E}{\partial \nu} & =\lambda_{0} E+F+\mu^{(1)} \psi_{0}^{(1)}+\mu^{(2)} \psi_{0}^{(2)}, \quad x \in \Sigma_{a},
\end{aligned}
$$

is solvable and the solution is orthogonal to the functions $\psi_{0}^{(i)}$ in $L_{2}\left(\Sigma_{a}\right)$ and $E \in \mathcal{A}$ and it has differentiable asymptotic expansion (4.4).

Similar to Corollary 1 but using Lemma 4.3 instead of Lemma 4.2 one can prove

Corollary 2. Let $\lambda_{0}$ be a double eigenvalue of Steklov problem (2.1), $\psi_{0}^{(1)}$ and $\psi_{0}^{(2)}$ be the associated eigenfunctions orthonormalized in $L_{2}\left(\Sigma_{a}\right)$ and satisfying (2.15). Then there exist 
functions $E_{q} \in \mathcal{A}, q=\overline{0, n}$, having differentiable asymptotic expansions (4.8), (4.9) as $r \rightarrow 0$ and solving the boundary value problems

$$
\begin{aligned}
-\Delta E_{q} & =0, \quad x \in \Pi \backslash\{0\}, \quad \mathfrak{l} E_{q}=0, \quad x \in \partial \Pi \backslash \bar{\Sigma}_{a}, \\
\frac{\partial E_{q}}{\partial \nu} & =\lambda_{0} E_{q}+\mu_{q}^{(1)} \psi_{0}^{(1)}+\mu_{q}^{(2)} \psi_{0}^{(2)}, \quad x \in \Sigma_{a},
\end{aligned}
$$

as

$$
\begin{array}{ll}
\mu_{0}^{(1)}=\left|S_{n}\right|(n-2) \psi_{0}^{(1)}(0), & \mu_{0}^{(2)}=0, \\
\mu_{m}^{(i)}=\left|S_{n}\right| \frac{\partial \psi_{0}^{(i)}}{\partial x_{m}}(0), & m=\overline{1, n}, \quad i=1,2 .
\end{array}
$$

In its true, Corollary 2 and Lemma 4.3 imply the following two statements.

Corollary 3. Let $\lambda_{0}$ be a double eigenvalue of Steklov problem (2.1), $\psi_{0}^{(1)}$ and $\psi_{0}^{(2)}$ be the associated eigenfunctions orthonormalized in $L_{2}\left(\Sigma_{a}\right)$ and satisfying (2.15). Then the function

$$
\widetilde{E}_{m}(x)=E_{m}(x)+\delta_{m} E_{1}(x) \in \mathcal{A}, \quad m=\overline{1, n},
$$

has the differentiable asymptotic expansion

$$
\widetilde{E}_{m}(x)=x_{m} r^{-n}+\delta_{m} r^{-n}+\sum_{k=0}^{\infty} X_{k}(x), \quad \delta_{m}=\frac{\frac{\partial \psi_{0}^{(2)}}{\partial x_{m}}(0)}{(n-2) \psi_{0}^{(1)}(0)}, \quad m=\overline{1, n},
$$

as $r \rightarrow 0$ and solves the boundary value problem

$$
\begin{aligned}
-\Delta \widetilde{E}_{m} & =0, \quad x \in \Pi \backslash\{0\}, \quad \mathfrak{l} \widetilde{E}_{m}=0, \quad x \in \partial \Pi \backslash \bar{\Sigma}_{a}, \\
\frac{\partial \widetilde{E}_{m}}{\partial \nu} & =\lambda_{0} \widetilde{E}_{m}+\mu_{m}^{(2)} \psi_{0}^{(2)}, \quad x \in \Sigma_{a},
\end{aligned}
$$

for $\mu_{m}^{(2)}$ determined by identity 4.16).

Lemma 4.4. Let $\lambda_{0}$ be a double eigenvalue of Steklov problem (2.1), $\psi_{0}^{(1)}$ and $\psi_{0}^{(2)}$ be the associated eigenfunction orthonormalized in $L_{2}\left(\Sigma_{a}\right), Y_{j}(x)$ be an arbitrary harmonic polynomial, $j \geqslant 1, F \in C^{\infty}\left(\bar{\Sigma}_{a}\right)$. Then there exists a function $\widetilde{E} \in \mathcal{A}$ orthogonal to the functions $\psi_{0}^{(i)}$ in $L_{2}\left(\Sigma_{a}\right)$ solving Steklov problem

$$
\begin{aligned}
-\Delta \widetilde{E} & =0, \quad x \in \Pi \backslash\{0\}, \quad l \widetilde{E}=0, \quad x \in \partial \Pi \backslash \bar{\Sigma}_{a}, \\
\frac{\partial \widetilde{E}}{\partial \nu} & =\lambda_{0} \widetilde{E}+F+\mu \psi_{0}^{(2)}, \quad x \in \Sigma_{a},
\end{aligned}
$$

and having differentiable asymptotic expansions:

$$
\widetilde{E}_{m}(x)=x_{m} r^{-n}+\delta r^{-n}+\sum_{k=0}^{\infty} X_{k}(x), \quad m=\overline{1, n}, \quad r \rightarrow 0,
$$

for some $\delta$ and $\mu$.

\section{Proof of Theorem 2.2}

Outside a neighbourhood of the cavity, it is natural to seek an approximation $U(x, \varepsilon)$ (external expansion) for the function $\psi_{\varepsilon}$ as $U(x, \varepsilon) \approx \psi_{0}(x)$. In the vicinity of $\omega_{\varepsilon}$, it is natural to seek an approximation $V(x, \varepsilon)$ (internal expansion) for the function $\psi_{\varepsilon}$ as an expansion in functions depending on the variable $\xi=x \varepsilon^{-1}$. 
Denote $\rho=|\xi|$. Rewriting the right hand side in (4.14) in terms of the variable $\xi$, we have

$$
U(x, \varepsilon) \approx \psi_{0}(x)=\psi_{0}(0)+\varepsilon \sum_{m=1}^{n} \frac{\partial \psi_{0}}{\partial x_{m}}(0) \xi_{m}+\sum_{k=2}^{\infty} \varepsilon^{k} X_{k}^{(0)}(\xi), \quad \rho \varepsilon=r \rightarrow 0 .
$$

Thus, following the method of matching asymptotic expansions [18], we seek the internal expansion as

$$
V(\xi, \varepsilon)=v_{0}(\xi)+\varepsilon v_{1}(\xi)+\sum_{k=2}^{\infty} \varepsilon^{k} v_{k}(\xi)
$$

where

$$
\begin{aligned}
& v_{0}(\xi) \sim X_{0}^{(0)}(\xi) \equiv \psi_{0}(0), \quad v_{1}(\xi) \sim X_{1}^{(0)}(\xi)=\sum_{m=1}^{n} \frac{\partial \psi_{0}}{\partial x_{m}}(0) \xi_{m}, \quad \rho \rightarrow \infty, \\
& v_{k}(\xi) \sim X_{k}^{(0)}(\xi), \quad k \geqslant 2, \quad \rho \rightarrow \infty
\end{aligned}
$$

Substituting (5.1) into (2.2), passing to the variable $\xi$ and equating the coefficients at the like powers of $\varepsilon$, we obtain the following boundary value problems for $v_{k}$

$$
\Delta_{\xi} v_{k}=0 \quad \xi \in \mathbb{R}^{n} \backslash \bar{\omega}, \quad v_{k}=0 \quad \xi \in \partial \omega .
$$

Remark 5.1. Here $\Delta_{\xi}$ stands for the Laplace operator in the variable $\xi$. Since below in the equations for the coefficients of the internal expansions the Laplace operator is used only in such sense, for the sake of simplicity we shall omit the subscript $\xi$ in $\Delta_{\xi}$.

The function

$$
v_{0}(\xi)=\psi_{0}(0)\left(1-z_{0}(\xi)\right)
$$

is a solution to boundary value problem (5.3) having the asymptotic expansion

$$
\begin{aligned}
v_{0}(\xi) & =X_{0}^{(0)}+\sum_{i=0}^{\infty} \frac{Y_{i}^{(0)}(\xi)}{\rho^{2 i+n-2}}, \quad \rho \rightarrow \infty, \\
Y_{0}^{(0)} & =-\psi_{0}(0) c(\omega), \quad Y_{k}^{(0)}(\xi)=-\psi_{0}(0) Z_{k}^{(0)}(\xi), \quad k \geqslant 1,
\end{aligned}
$$

which specifies required asymptotic expansion $(5.2)$ for $v_{0}(\xi)$.

Rewriting (5.5) in the variables $x=\varepsilon \xi$, we obtain that

$$
v_{0}(\xi)=X_{0}^{(0)}+\sum_{i=0}^{\infty} \varepsilon^{n-2+i} \frac{Y_{i}^{(0)}(x)}{r^{2 i+n-2}}, \quad \varepsilon^{-1} r \rightarrow \infty .
$$

In view of this identity and in accordance with the method of matching asymptotic expansions, the external expansion for the eigenfunction should be sought as

$$
U(x, \varepsilon)=\psi_{0}(x)+\varepsilon^{n-2} \sum_{i=0}^{\infty} \varepsilon^{i} \psi_{i+n-2}(x),
$$

where

$$
\begin{aligned}
\psi_{n-2}(x) & \sim Y_{0}^{(0)} r^{-n+2}=-\psi_{0}(0) c(\omega) r^{-n+2}, \quad r \rightarrow 0, \\
\psi_{i+n-2}(x) & \sim Y_{i}^{(0)} r^{-n-2 i+2}=-\psi_{0}(0) Z_{i}^{(0)}(x) r^{-2 i-n+2}, \quad i \geqslant 1, \quad r \rightarrow 0 .
\end{aligned}
$$

Since the external expansion should describe the behavior of the eigenfunction almost in the entire domain $\Pi$ (except a small neighbourhood of the hole), by analogy with $(5.8)$, it is natural to seek the asymptotic expansion for the eigenvalue as series (2.7). 
Substituting series $(5.8)$ and $(2.7)$ into $(2.2)$ and equating the coefficients at the like powers of $\varepsilon$, we obtain boundary value problem 2.1) for $\psi_{0}$ and the following boundary value problems for other coefficients of external expansion (5.8):

$$
\begin{aligned}
& -\Delta \psi_{n-2}=0, \quad x \in \Pi \backslash\{0\}, \quad \mathfrak{l} \psi_{n-2}=0, \quad x \in \partial \Pi \backslash \bar{\Sigma}_{a}, \\
& \frac{\partial \psi_{n-2}}{\partial \nu}=\lambda_{0} \psi_{n-2}+\lambda_{n-2} \psi_{0}, \quad x \in \Sigma_{a}, \\
& -\Delta \psi_{n-2+i}=0, \quad x \in \Pi \backslash\{0\}, \quad \mathfrak{l} \psi_{n-2+i}=0, \quad x \in \partial \Pi \backslash \bar{\Sigma}_{a}, \\
& \frac{\partial \psi_{n-2+i}}{\partial \nu}=\lambda_{0} \psi_{n-2+i}+\lambda_{n-2+i} \psi_{0}, \quad x \in \Sigma_{a}, \quad 1 \leqslant i \leqslant n-3, \\
& -\Delta \psi_{n-2+i}=0, \quad x \in \Pi \backslash\{0\}, \quad \mathfrak{l} \psi_{n-2+i}=0, \quad x \in \partial \Pi \backslash \bar{\Sigma}_{a}, \\
& \frac{\partial \psi_{n-2+i}}{\partial \nu}=\lambda_{0} \psi_{n-2+i}+\lambda_{n-2+i} \psi_{0}+\sum_{k=0}^{i-n+2} \lambda_{n-2+k} \psi_{i-k}, \quad x \in \Sigma_{a}, \quad i \geqslant n-2 .
\end{aligned}
$$

By Corollary 1, the function

$$
\psi_{n-2}(x)=-\psi_{0}(0) c(\omega) E_{0}(x) \in \mathcal{A},
$$

solves boundary value problem (5.11) for $\lambda_{n-2}$ determined by identity (2.8) and has the differentiable asymptotic expansion

$$
\psi_{n-2}(x)=Y_{0}^{(0)} r^{-n+2}+\sum_{j=0}^{\infty} X_{j}^{(n-2)}(x), \quad r \rightarrow 0,
$$

which specifies asymptotics 5.9 .

Remark 5.2. Thus, we have proved the existence of functions $v_{0}(\xi)$ and $\psi_{n-2}(x)$ solving boundary value problems (5.3) and (5.11) for $\lambda_{n-2}$ defined by identity (2.8) and having asymptotics (5.2) and (5.9).

It is easy to see that in the class of functions orthogonal to $\psi_{0}$ in $L_{2}\left(\Sigma_{a}\right)$, the solution $\psi_{n-2}(x)$ to boundary value problem (5.11) with asymptotics (5.9) is unique. However, it is also easy to see that the solutions to problems (5.12) and (5.13) are not uniquely determined by the main singularities (5.10) at zero; for instance, we can add the term $\alpha E_{0}(x)$ with arbitrary $\alpha$.

In the same way, it is easy to see that the unique solution $v_{0}(\xi)$ to boundary value problem (5.3) with asymptotics (5.2) is determined by identity (5.4). On the other hand, it is also easy to see that as $k \geqslant 1$, the solutions $v_{k}(\xi)$ to boundary value problems (5.3) with asymptotics (5.2) at infinity are determined non-uniquely; for instance, we can add the term $\alpha z_{0}(x)$ with an arbitrary $\alpha$.

Thus, we have constructed the leading terms $v_{0}(\xi), \psi_{n-2}(x)$ and $\lambda_{n-2}$ for asymptotic expansions (5.1), (5.8) and (2.7) and we have determined the leading terms of the asymptotics at infinity and at zero for the coefficients $v_{k}(\xi)$ and $\psi_{n-2+k}(x)$ as $k \geqslant 1$, respectively.

The matter of the further matching of series (5.1) and (5.8) is constructing the solutions $v_{k}(\xi)$ and $\psi_{n-2+k}(x)$ to boundary value problems (5.3) and (5.12), (5.13) such that if in (5.8) we replace the coefficients $\psi_{0}(x)$ and $\psi_{n-2+i}(x)$ by their asymptotic expansion as $r \rightarrow 0$ and pass to the variable $\xi=\varepsilon^{-1} x$ and if in series (5.1) we replace the coefficients $v_{i}(\xi)$ by their asymptotic expansions as $\rho \rightarrow \infty$, then we obtain to same series.

The following statement is a key one for matching the asymptotic expansions.

Lemma 5.1. Let

$$
\Psi^{\varepsilon}(x)=\Psi_{0}(x)+\varepsilon^{n-2} \sum_{i=0}^{\infty} \varepsilon^{i} \Psi_{i+n-2}(x),
$$




$$
\begin{aligned}
& \Phi^{\varepsilon}(\xi)=\sum_{i=0}^{\infty} \varepsilon^{i} \Phi_{i}(\xi) \\
& \Psi_{0}(x)=\sum_{k=0}^{\infty} X_{k}^{(0)}(x) \\
& \Psi_{n-2+i}(x)=\sum_{k=0}^{i} Y_{k}^{(i-k)}(x) r^{-2 k-n+2}+\sum_{k=0}^{\infty} X_{k}^{(n-2+i)}(x), \quad i \geqslant 0, \\
& \Phi_{i}(\xi)=X_{i}^{(0)}(\xi)+\sum_{k=0}^{\infty} Y_{k}^{(i)}(\xi) \rho^{-2 k-n+2}, \quad 0 \leqslant i<n-2, \\
& \Phi_{i}(\xi)=X_{i}^{(0)}(\xi)+\sum_{k=0}^{i-n+2} X_{k}^{(i-k)}(\xi)+\sum_{k=0}^{\infty} Y_{k}^{(i)}(\xi) \rho^{-2 k-n+2}, \quad i \geqslant n-2,
\end{aligned}
$$

where $X_{q}^{(j)}, Y_{q}^{(j)}$ are arbitrary harmonic polynomials. Then

$$
\Psi^{\varepsilon}(\varepsilon \xi)=\Phi^{\varepsilon}(\xi) .
$$

There exist $v_{i} \in C^{\infty}\left(\mathbb{R}^{n} \backslash \bar{\omega}\right), \psi_{n-2+i} \in \mathcal{A}, i \geqslant 0$, satisfying boundary value problems (5.3) and (5.11), (5.12), (5.13) for some $\lambda_{n-2+i}$ and having asymptotic expansions

$$
\begin{aligned}
v_{i}(\xi) & =\Phi_{i}(\xi), \quad \rho \rightarrow \infty, \\
\psi_{n-2+i}(x) & =\Psi_{n-2+i}(x), \quad r \rightarrow 0,
\end{aligned}
$$

where $X_{q}^{(0)}$ come from expansion (4.14) at zero for the function $\psi_{0}(x)$, while other $X_{q}^{(j)}, Y_{q}^{(j)}$ are some harmonic polynomials.

Identities (2.8), (5.4) and (5.14) hold true.

If $\psi_{0}(0)=0$, then identities (2.9), (2.10), (2.11) hold true and

$$
\begin{aligned}
v_{0}(\xi) & \equiv 0, \quad \psi_{n-2}(x) \equiv 0, \\
v_{1}(\xi) & =\sum_{m=1}^{n} \frac{\partial \psi_{0}}{\partial x_{m}}(0)\left(\xi_{m}-z_{m}(\xi)\right), \\
\psi_{n-1}(x) & =-E_{0}(x) \sum_{m=1}^{n} \frac{\partial \psi_{0}}{\partial x_{m}}(0) c_{m, 0} .
\end{aligned}
$$

Proof. Identity (5.22) is checked by changing $x=\varepsilon \xi$ in $\Psi^{\varepsilon}(x)$.

The statements of the lemma for $\lambda_{n-2}, v_{0}(\xi)$ and $\psi_{n-2}(x)$ including identities (2.8), (5.4) and (5.14) have already been proved. We stress that since the functions $\psi_{0}(x), v_{0}(\xi)$ and $\psi_{n-2}(x)$ are found, then the same is true for the harmonic polynomials $X_{k}^{(0)}, Y_{k}^{(0)}$ and $X_{k}^{(n-2)}, k \geqslant 0$.

It follows from the definition of $\Phi_{1}(\xi)$ that

$$
\begin{aligned}
& \Phi_{1}(\xi)=X_{1}^{(0)}(\xi)+X_{0}^{(1)}+\sum_{k=0}^{\infty} Y_{k}^{(1)}(\xi) \rho^{-2 k-1}, \quad n=3, \\
& \Phi_{1}(\xi)=X_{1}^{(0)}(\xi)+\sum_{k=0}^{\infty} Y_{k}^{(1)}(\xi) \rho^{-2 k-n+2}, \quad n>3,
\end{aligned}
$$

where

$$
X_{1}^{(0)}(\xi)=\sum_{m=1}^{n} \frac{\partial \psi_{0}}{\partial x_{m}}(0) \xi_{m}
$$


in accordance with 4.14), $X_{0}^{(1)}$ is the determined constant from $\Psi_{n-1}$ for $n=3$, and $Y_{k}^{(1)}$ are still arbitrary harmonic polynomials. It follows from the definition of the functions $z_{q}$ (see (2.5)) that the function

$$
\begin{aligned}
& v_{1}(\xi)=\sum_{m=1}^{n} \frac{\partial \psi_{0}}{\partial x_{m}}(0)\left(\xi_{m}-z_{m}(\xi)\right)+X_{0}^{(1)}\left(1-z_{0}(\xi)\right), \quad n=3, \\
& v_{1}(\xi)=\sum_{m=1}^{n} \frac{\partial \psi_{0}}{\partial x_{m}}(0)\left(\xi_{m}-z_{m}(\xi)\right), \quad n>3
\end{aligned}
$$

is a solution to boundary value problem (5.3) having asymptotic expansions (5.23), (5.20), 5.21 , 5.28, 5.29 with some harmonic polynomials $Y_{k}^{(1)}(\xi)$ and

$$
\begin{aligned}
& Y_{0}^{(1)}(\xi)=-\sum_{m=1}^{n} \frac{\partial \psi_{0}}{\partial x_{m}}(0) c_{m, 0}-c(\omega) X_{0}^{(1)}, \quad n=3, \\
& Y_{0}^{(1)}(\xi)=-\sum_{m=1}^{n} \frac{\partial \psi_{0}}{\partial x_{m}}(0) c_{m, 0}, \quad n>3 \\
& Y_{1}^{(1)}(\xi)=-\sum_{m=1}^{n} \frac{\partial \psi_{0}}{\partial x_{m}}(0) \sum_{q=1}^{n} c_{m, q} \xi_{q}-X_{0}^{(1)} \sum_{q=1}^{n} c_{0, q} \xi_{q}, \quad n=3, \\
& Y_{1}^{(1)}(\xi)=-\sum_{m=1}^{n} \frac{\partial \psi_{0}}{\partial x_{m}}(0) \sum_{q=1}^{n} c_{m, q} \xi_{q}, \quad n>3 .
\end{aligned}
$$

Having determined $Y_{k}^{(1)}(\xi)$, by Lemma 4.2 we obtain that there exists a function $\psi_{n-1} \in \mathcal{A}$ solving problem (5.13) for $n=3$ and problem (5.12) for $n \geqslant 4$ for some $\lambda_{n-1}$ and having asymptotic expansion 5.24 for some harmonic polynomials $X_{k}^{(n-1)}(x)$.

Then, having determined $X_{k}^{(n-1)}(x)$, by Lemma 4.1 we obtain that there exists a solution $v_{2} \in C^{\infty}\left(\mathbb{R}^{n} \backslash \bar{\omega}\right)$ to boundary value problem (5.3) having asymptotic expansion $(5.23)$ for some harmonic polynomials $Y_{k}^{(2)}(\xi)$.

In its turn, having determined $Y_{k}^{(2)}(\xi)$, by Lemma 4.2 we obtain that there exist a constant $\lambda_{n}$ and a function $\psi_{n} \in \mathcal{A}$ such that $\psi_{n}$ is a solution to problem (5.13) as $n=4$ and to problem 5.12 as $n \geqslant 5$ and has asymptotic expansion 5.24 for some harmonic polynomials $X_{k}^{(n)}(x)$ and so forth.

Let $\psi_{0}(0)=0$. Then by (5.4), (5.14) and (2.8) we get identities (5.25), (2.9). Therefore, first,

$$
\begin{aligned}
Y_{1}^{(0)}(\xi) & \equiv 0, \\
Y_{2}^{(0)}(\xi) & \equiv 0,
\end{aligned}
$$

and, second, $X_{0}^{(1)}=0$ as $n=3$ and by 5.32, 5.33, 5.34, 5.35 we obtain that

$$
\begin{aligned}
Y_{0}^{(1)} & =-\sum_{m=1}^{n} \frac{\partial \psi_{0}}{\partial x_{m}}(0) c_{m, 0}, \quad n \geqslant 3, \\
Y_{1}^{(1)}(\xi) & =-\sum_{m=1}^{n} \frac{\partial \psi_{0}}{\partial x_{m}}(0) \sum_{q=1}^{n} c_{m, q} \xi_{q}, \quad n \geqslant 3 .
\end{aligned}
$$

Since $\psi_{0}(0)=\lambda_{n-2}=0$, the function $\psi_{n-1}(x)$, determined by identity (5.27) has asymptotic expansion (5.24), (5.19), (5.36), (5.38) as $i=1$. Thus, in accordance with Corollary 1, it solves boundary value problem (5.12) as $n \geqslant 4$ and boundary value problem (5.13) as $n=3$ for $\lambda_{n-1}=$ 
0. That is, identity 2.10 holds true. In its turn, since $\psi_{0}(0)=\psi_{n-2}(x)=\lambda_{n-2}=\lambda_{n-1}=0$, the function

$$
\psi_{n}(x)=-\sum_{m=1}^{n} \frac{\partial \psi_{0}}{\partial x_{m}}(0) \sum_{q=1}^{n} c_{m, q} E_{q}(x)+Y_{0}^{(2)} E_{0}(x)
$$

has asymptotic expansion (5.24), (5.19), (5.37), (5.39) for $i=2$ and in accordance with Corollary 1 is solves boundary value problem $(5.12)$ as $n \geqslant 4$ and boundary value problem (5.13) as $n=3$ for $\lambda_{n}$ determined by identity (2.11).

We denote $\widehat{\lambda}_{\varepsilon, N}, \widehat{u}_{\varepsilon, N}(x), \widehat{v}_{\varepsilon, N}(\xi)$ partial sums of series (2.7), (5.8) and (5.1) up to power $N$. Lemma 5.1 implies the following statement.

Lemma 5.2. The function $\widehat{u}_{\varepsilon, N} \in \mathcal{A}$ solves the boundary value problem

$$
\begin{aligned}
-\Delta \widehat{u}_{\varepsilon, N}(x) & =0, \quad x \in \Pi \backslash\{0\}, \quad \mathfrak{l} \widehat{u}_{\varepsilon, N}(x)=0, \quad x \in \partial \Pi \backslash \bar{\Sigma}_{a}, \\
\frac{\partial \widehat{u}_{\varepsilon, N}(x)}{\partial \nu} & =\widehat{\lambda}_{\varepsilon, N} \widehat{u}_{\varepsilon, N}(x)+O\left(\varepsilon^{N+1}\right), \quad x \in \Sigma_{a} .
\end{aligned}
$$

The convergence holds:

$$
\int_{\Sigma_{a}}\left(\widehat{u}_{\varepsilon, N}-\psi_{\varepsilon}\right)^{2} d x^{\prime} \rightarrow 0, \quad \varepsilon \rightarrow 0 .
$$

The function $\widehat{v}_{\varepsilon, N} \in C^{\infty}\left(\mathbb{R}^{n} \backslash \bar{\omega}\right)$ solves the boundary value problem

$$
\Delta_{\xi} \widehat{v}_{\varepsilon, N}(\xi)=0, \quad \xi \in \mathbb{R}^{n} \backslash \bar{\omega}, \quad \widehat{v}_{\varepsilon, N}(\xi)=0, \quad \xi \in \partial \omega .
$$

As $\varepsilon^{\frac{1}{2}}<r<2 \varepsilon^{\frac{1}{2}}$ (or the same, as $\varepsilon^{-\frac{1}{2}}<\rho<2 \varepsilon^{-\frac{1}{2}}$ ) the differentiable identity holds:

$$
\widehat{u}_{\varepsilon, N}(x)-\widehat{v}_{\varepsilon, N}(\xi)=O\left(r^{N+1}+\varepsilon^{N} r+\rho^{-N-1}+\varepsilon^{N} \rho^{-1}\right) .
$$

We denote

$$
\widetilde{u}_{\varepsilon, N}(x)=\tilde{\chi}\left(r \varepsilon^{-\frac{1}{2}}\right) \widehat{u}_{\varepsilon, N}(x)+\left(1-\tilde{\chi}\left(r \varepsilon^{-\frac{1}{2}}\right)\right) \widehat{v}_{\varepsilon, N}\left(\frac{x}{\varepsilon}\right),
$$

where, we recall, $\tilde{\chi}(t)$ is an infinitely differentiable cut-off function vanishing as $t \leqslant 1$ and equalling to one as $t \geqslant 2$.

It follows from 5.40 and Theorem 2.1 that

$$
\int_{\Sigma_{a}} \widetilde{u}_{\varepsilon, N} \psi_{\varepsilon} d x^{\prime} \rightarrow 1, \quad \varepsilon \rightarrow 0 .
$$

Lemma 5.3. The function $\widetilde{u}_{\varepsilon, N} \in H^{1}\left(\Pi_{\varepsilon}\right)$ is a solution to the boundary value problem

$$
\begin{aligned}
& -\Delta \widetilde{u}_{\varepsilon, N}=F_{\varepsilon, N}, \quad x \in \Pi_{\varepsilon}, \quad \mathfrak{u}_{\varepsilon, N}=0, \quad x \in \partial \Pi \backslash \bar{\Sigma}_{a}, \\
& \frac{\partial \widetilde{u}_{\varepsilon, N}}{\partial \nu}=\widehat{\lambda}_{\varepsilon, N} \widetilde{u}_{\varepsilon, N}+g_{\varepsilon, N}, \quad x \in \Sigma_{a}, \quad \widetilde{u}_{\varepsilon, N}=0, \quad x \in \partial \omega_{\varepsilon},
\end{aligned}
$$

where

$$
\begin{aligned}
& \left\|g_{\varepsilon, N}\right\|_{L_{2}\left(\Sigma_{a}\right)} \leqslant C \varepsilon^{N+1}, \\
& \left\|F_{\varepsilon, N}\right\|_{L_{2}\left(\Pi_{\varepsilon}\right)} \leqslant C \varepsilon^{\frac{2 N+n-2}{4}}, \\
& \operatorname{supp} F_{\varepsilon, N} \subset \bar{B}_{2 \varepsilon^{\frac{1}{2}}} \backslash B_{\varepsilon^{\frac{1}{2}}} .
\end{aligned}
$$

Proof. All statements except (5.45) and (5.46) are implied directly by Lemma 5.2. By applying the Laplace operator to the function $\widetilde{u}_{\varepsilon, N}$, we obtain that

$$
F_{\varepsilon, N}(x)=-\left(\widehat{u}_{\varepsilon, N}\left(\frac{x}{\varepsilon}\right)-\widehat{v}_{\varepsilon, N}(x)\right) \Delta \widetilde{\chi}\left(r \varepsilon^{-\frac{1}{2}}\right)-\nabla\left(\widehat{u}_{\varepsilon, N}\left(\frac{x}{\varepsilon}\right)-\widehat{v}_{\varepsilon, N}(x)\right) \nabla \widetilde{\chi}\left(r \varepsilon^{-\frac{1}{2}}\right) .
$$

This yields (5.46). In its turn, (5.47), (5.46) and (5.41) imply estimate (5.45). 
Multiplying equation $(5.43)$ by the eigenfunction $\psi_{\varepsilon}$ and integrating by parts over $\Pi_{\varepsilon}$, thanks to boundary value problems (5.43), 2.2 we obtain

$$
\left(\lambda_{\varepsilon}-\widehat{\lambda}_{\varepsilon, N}\right) \int_{\Sigma_{a}} \widetilde{u}_{\varepsilon, N} \psi_{\varepsilon} d x^{\prime}=\int_{\Pi_{\varepsilon}} F_{\varepsilon, N} \psi_{\varepsilon} d x+\int_{\Sigma_{a}} g_{\varepsilon, N} \psi_{\varepsilon} d x^{\prime} .
$$

Since $\left\|\psi_{\varepsilon}\right\|_{L_{2}\left(\Sigma_{a}\right)}=1$, by 5.44 we obtain that

$$
\int_{\Sigma_{a}} g_{\varepsilon, N} \psi_{\varepsilon} d x^{\prime}=O\left(\varepsilon^{N+1}\right) .
$$

By the integral identity for the function $\psi_{\varepsilon}$ we have:

$$
\int_{\Pi_{\varepsilon}}\left|\nabla \psi_{\varepsilon}\right|^{2} d x+\widetilde{h} \int_{\partial \Pi_{\varepsilon} \backslash \bar{\Sigma}_{a}} \psi_{\varepsilon}^{2} d s=\lambda_{\varepsilon}
$$

where $\widetilde{h}=0$ if $H=0$ and $\widetilde{h}=\frac{h}{H}$ if $H \neq 0$. Therefore,

$$
\left\|\psi_{\varepsilon}\right\|_{H^{1}\left(\Pi_{\varepsilon}\right)}^{2}=\int_{\Pi_{\varepsilon}}\left|\nabla \psi_{\varepsilon}\right|^{2} d x+\int_{\Sigma_{a}} \psi_{\varepsilon}^{2} d x^{\prime} \leqslant C .
$$

Then, extending $\psi_{\varepsilon}$ by zero in $\omega_{\varepsilon}$, by 3.14 we get $\left\|\psi_{\varepsilon}\right\|_{W_{2}^{1}(\Pi(R))}^{2} \leqslant C$. This is why it follows from (5.45) and 5.46) that

$$
\int_{\Pi_{\varepsilon}} F_{\varepsilon, N} \psi_{\varepsilon} d x=O\left(\varepsilon^{\frac{2 N+n-2}{4}}\right) .
$$

It follows from (5.48), (5.49), (5.50) and (5.42) that

$$
\lambda_{\varepsilon}-\widehat{\lambda}_{\varepsilon, N}=O\left(\varepsilon^{\frac{2 N+n-2}{4}}\right) .
$$

Since $N$ is arbitrary, it proves (2.7). The proof of Theorem 2.2 is complete.

\section{Proof of Theorem 2.3}

Hereinafter $\lambda_{0}$ is a double eigenvalue of Steklov problem 2.1 , and $\psi_{0}^{(1)}(x)$ and $\psi_{0}^{(2)}(x)$ are the associated orthonormalized in $L_{2}\left(\Sigma_{a}\right)$ eigenfunctions chosen in accordance with (2.15). The Taylor series for functions $\psi_{0}^{(s)}(x)$ at zero are of the form:

$$
\begin{aligned}
\psi_{0}^{(1)}(x) & =\sum_{k=0}^{\infty} X_{k}^{(0,1)}(x), \quad \psi_{0}^{(2)}(x)=\sum_{k=1}^{\infty} X_{k}^{(0,2)}(x), \quad r \rightarrow 0, \\
X_{0}^{(0,1)}(x) & =\psi_{0}^{(1)}(0), \quad X_{1}^{(0, s)}(x)=\sum_{m=1}^{n} \frac{\partial \psi_{0}^{(s)}}{\partial x_{m}}(0) x_{m} .
\end{aligned}
$$

Outside a neighbourhood of the cavity, we construct the external expansion $U^{(1)}(x, \varepsilon)$ for the eigenfunction $\psi_{\varepsilon}^{(1)}(x)$ for Steklov problem 2.2 as $U^{(1)}(x, \varepsilon) \approx \psi_{0}^{(1)}(x)$. Rewriting 6.1 in the variable $\xi$, we have

$$
U^{(1)}(x, \varepsilon) \approx \psi_{0}^{(1)}(x)=\psi_{0}^{(1)}(0)+\varepsilon \sum_{m=1}^{n} \frac{\partial \psi_{0}^{(1)}}{\partial x_{m}}(0) \xi_{m}+\sum_{k=2}^{\infty} \varepsilon^{k} X_{k}^{(0,1)}(\xi), \quad \rho \varepsilon=r \rightarrow 0 .
$$

Following the matching method, we construct the internal expansion as

$$
V^{(1)}(\xi, \varepsilon)=v_{0}^{(1)}(\xi)+\varepsilon v_{1}^{(1)}(\xi)+\sum_{k=2}^{\infty} \varepsilon^{k} v_{k}^{(1)}(\xi)
$$


where

$$
\begin{aligned}
& v_{0}^{(1)}(\xi) \sim X_{0}^{(0,1)}(\xi) \equiv \psi_{0}^{(1)}(0), \quad \rho \rightarrow \infty, \\
& v_{1}^{(1)}(\xi) \sim X_{1}^{(0,1)}(\xi)=\sum_{m=1}^{n} \frac{\partial \psi_{0}^{(1)}}{\partial x_{m}}(0) \xi_{m}, \quad \rho \rightarrow \infty, \\
& v_{k}^{(1)}(\xi) \sim X_{k}^{(0,1)}(\xi), \quad k \geqslant 2, \quad \rho \rightarrow \infty .
\end{aligned}
$$

Substituting (6.2) into (2.2), passing to the variable $\xi$ and equating the coefficients at the like powers of $\varepsilon$, we obtain the boundary value problems for $v_{k}^{(1)}$ :

$$
\Delta_{\xi} v_{k}^{(1)}=0 \quad \xi \in \mathbb{R}^{n} \backslash \bar{\omega}, \quad v_{k}^{(1)}=0 \quad \xi \in \partial \omega .
$$

The function

$$
v_{0}^{(1)}(\xi)=\psi_{0}^{(1)}(0)\left(1-z_{0}(\xi)\right)
$$

is a solution to boundary value problem (6.4) having the differentiable asymptotic expansion

$$
\begin{aligned}
v_{0}^{(1)}(\xi) & =X_{0}^{(0,1)}+\sum_{i=0}^{\infty} \frac{Y_{i}^{(0,1)}(\xi)}{\rho^{2 i+n-2}}, \quad \rho \rightarrow \infty, \\
Y_{0}^{(0,1)} & =-\psi_{0}^{(1)}(0) c(\omega), \quad Y_{k}^{(0,1)}(\xi)=-\psi_{0}^{(1)}(0) Z_{k}^{(0,1)}(\xi), \quad k \geqslant 1,
\end{aligned}
$$

which specifies required asymptotics 6.3 for $v_{0}^{(1)}(\xi)$.

Rewriting now (6.6) in variables $x=\varepsilon \xi$, we obtain that

$$
v_{0}^{(1)}(\xi)=X_{0}^{(0,1)}+\sum_{i=0}^{\infty} \varepsilon^{n-2+i} \frac{Y_{i}^{(0,1)}(x)}{r^{2 i+n-2}}, \quad \varepsilon^{-1} r \rightarrow \infty .
$$

In view of this identity, similar to the previous section one can suggest to seek the external expansion for the eigenfunction as

$$
U^{(1)}(x, \varepsilon)=\psi_{0}^{(1)}(x)+\varepsilon^{n-2} \sum_{i=0}^{\infty} \varepsilon^{i} \psi_{i+n-2}^{(1)}(x),
$$

where

$$
\begin{aligned}
\psi_{n-2}^{(1)}(x) & \sim Y_{0}^{(0,1)} r^{-n+2}=-\psi_{0}^{(1)}(0) c(\omega) r^{-n+2}, \quad r \rightarrow 0, \\
\psi_{j+n-2}^{(1)}(x) & \sim Y_{j}^{(0,1)}(x) r^{-n-2 j+2}, \quad r \rightarrow 0, \quad j \geqslant 1 .
\end{aligned}
$$

But since in the considered case of the double eigenvalue $\lambda_{0}$ there is one more eigenfunction $\psi_{0}^{(2)}(x)$, we construct the external expansion as

$$
U^{(1)}(x, \varepsilon)=\psi_{0}^{(1)}(x)+\varepsilon^{n-2} \sum_{i=0}^{\infty} \varepsilon^{i} \psi_{i+n-2}^{(1)}(x)+\varepsilon \psi_{0}^{(2)}(x) \sum_{i=0}^{\infty} \alpha_{i+1}^{(1)} \varepsilon^{i},
$$

where the coefficients $\psi_{i+n-2}^{(1)}(x)$ have asymptotics $(6.9),(6.10)$, and $\alpha_{i+1}^{(1)}$ are some constants.

Similar to 2.7), we seek the asymptotic expansion for eigenvalue $\lambda_{\varepsilon}^{(1)}$ as $(2.16)$.

Substituting series (6.11) and (2.16) into (2.2), we obtain boundary value problems for the coefficients of external expansion (6.11):

$$
\begin{aligned}
-\Delta \psi_{n-2}^{(1)} & =0, \quad x \in \Pi \backslash\{0\}, \quad \mathfrak{l} \psi_{n-2}^{(1)}=0, \quad x \in \partial \Pi \backslash \bar{\Sigma}_{a}, \\
\frac{\partial \psi_{n-2}^{(1)}}{\partial \nu} & =\lambda_{0} \psi_{n-2}^{(1)}+\lambda_{n-2}^{(1)} \psi_{0}^{(1)}, \quad x \in \Sigma_{a},
\end{aligned}
$$




$$
\begin{aligned}
-\Delta \psi_{n-2+i}^{(1)}= & 0, \quad x \in \Pi \backslash\{0\}, \quad \mathfrak{l} \psi_{n-2+i}^{(1)}=0, \quad x \in \partial \Pi \backslash \bar{\Sigma}_{a}, \\
\frac{\partial \psi_{n-2+i}^{(1)}}{\partial \nu}= & \lambda_{0} \psi_{n-2+i}^{(1)}+\psi_{0}^{(2)} \sum_{p=1}^{i-1} \alpha_{p}^{(1)} \lambda_{i+n-2-p}^{(1)} \\
& +\lambda_{n-2+i}^{(1)} \psi_{0}^{(1)}+\alpha_{i}^{(1)} \lambda_{n-2}^{(1)} \psi_{0}^{(2)}, \quad x \in \Sigma_{a}, 1 \leqslant i \leqslant n-3, \\
-\Delta \psi_{n-2+i}^{(1)}= & 0, \quad x \in \Pi \backslash\{0\}, \quad \mathfrak{l} \psi_{n-2+i}^{(1)}=0, \quad x \in \partial \Pi \backslash \bar{\Sigma}_{a}, \\
\frac{\partial \psi_{n-2+i}^{(1)}}{\partial \nu}= & \lambda_{0} \psi_{n-2+i}^{(1)}+\sum_{k=0}^{i-n+2} \lambda_{n-2+k}^{(1)} \psi_{i-k}^{(1)}+\psi_{0}^{(2)} \sum_{p=1}^{i-1} \alpha_{p}^{(1)} \lambda_{i+n-2-p}^{(s)}+ \\
& +\lambda_{n-2+i}^{(1)} \psi_{0}^{(1)}+\alpha_{i}^{(1)} \lambda_{n-2}^{(1)} \psi_{0}^{(2)}, \quad x \in \Sigma_{a}, i \geqslant n-2 .
\end{aligned}
$$

By Corollary 2, the function

$$
\psi_{n-2}^{(1)}(x)=-\psi_{0}^{(1)}(0) c(\omega) E_{0}(x), \quad \psi_{n-2}^{(1)} \in \mathcal{A},
$$

is a solution to boundary value problem 6.12 for $\lambda_{n-2}^{(1)}$ determined by identity $(2.18$ ) and it has the differentiable asymptotic expansion:

$$
\psi_{n-2}^{(1)}(x)=Y_{0}^{(0,1)} r^{-n+2}+\sum_{j=0}^{\infty} X_{j}^{(n-2,1)}(x), \quad r \rightarrow 0,
$$

which specifies required asymptotics (6.9).

Remark 6.1. Thus, we have proved the existence of the functions $v_{0}^{(1)}(\xi)$ and $\psi_{n-2}^{(1)}(x)$ being solutions to boundary value problems (6.4) and (6.12) for $\lambda_{n-2}^{(1)}$ determined by identity (2.18) and having asymptotics (6.3) and (6.9).

Lemma 6.1. Let

$$
\begin{aligned}
& \Psi^{\varepsilon, 1}(x)= \Psi_{0}^{(1)}(x)+\varepsilon^{n-2} \sum_{i=0}^{\infty} \varepsilon^{i} \Psi_{i+n-2}^{(1)}(x)+\varepsilon \Psi_{0}^{(2)}(x) \sum_{i=0}^{\infty} \alpha_{i+1}^{(1)} \varepsilon^{i}, \\
& \Psi_{0}^{(1)}(x)=\sum_{k=0}^{\infty} X_{k}^{(0,1)}(x), \quad \Psi_{0}^{(2)}(x)=\sum_{k=1}^{\infty} X_{k}^{(0,2)}(x), \\
& \Psi_{n-2+i}^{(1)}(x)=\sum_{k=0}^{i} Y_{k}^{(i-k .1)}(x) r^{-2 k-n+2}+\sum_{k=0}^{\infty} X_{k}^{(n-2+i, 1)}(x), \quad i \geqslant 0, \\
& \Phi^{\varepsilon, 1}(\xi)=\sum_{i=0}^{\infty} \varepsilon^{i} \Phi_{i}^{(1)}(\xi), \\
& \Phi_{i}^{(1)}(\xi)=X_{i}^{(0,1)}(\xi)+\sum_{k=0}^{\infty} Y_{k}^{(i, 1)}(\xi) \rho^{-2 k-n+2}+\sum_{k=0}^{i-2} \alpha_{k+1}^{(1)} X_{i-k-1}^{(0,2)}(\xi), \quad 0 \leqslant i<n-2, \\
& \Phi_{i}^{(1)}(\xi)=X_{i}^{(0,1)}(\xi)+\sum_{k=0}^{i-n+2} X_{k}^{(i-k, 1)}(\xi)+\sum_{k=0}^{\infty} Y_{k}^{(i, 1)}(\xi) \rho^{-2 k-n+2}+ \\
& \quad+\sum_{k=0}^{i-2} \alpha_{k+1}^{(1)} X_{i-k-1}^{(0,2)}(\xi), \quad i \geqslant n-2,
\end{aligned}
$$

where $X_{q}^{(j, p)}, Y_{q}^{(j, 1)}$ are arbitrary harmonic polynomials and $\alpha_{j}^{(1)}$ are arbitrary numbers. Then

$$
\Psi^{\varepsilon, 1}(\varepsilon \xi)=\Phi^{\varepsilon, 1}(\xi) .
$$


There exist $v_{i}^{(1)} \in C^{\infty}\left(\mathbb{R}^{n} \backslash \bar{\omega}\right), \psi_{n-2+i}^{(1)} \in \mathcal{A}, i \geqslant 0$, satisfying boundary value problems (6.4) and 6.12), 6.13), 6.14) for some $\lambda_{n-2+i}^{(1)}$ and having differentiable asymptotic expansions

$$
\begin{aligned}
v_{i}^{(1)}(\xi) & =\Phi_{i}^{(1)}(\xi), \quad \rho \rightarrow \infty, \\
\psi_{n-2+i}^{(1)}(x) & =\Psi_{n-2+i}^{(1)}(x), \quad r \rightarrow 0,
\end{aligned}
$$

where $X_{q}^{(0, s)}$ come from the expansion at zero 6.1$)$ for the functions $\psi_{0}^{(s)}(x)$, other $X_{q}^{(j, 1)}, Y_{q}^{(j, 1)}$ are some harmonic polynomials, and $\alpha_{j}^{(1)}$ are some numbers.

Identities (2.18), (6.5) and (6.15) hold.

Proof. Identity 6.19 is checked by making the change $x=\varepsilon \xi$ in $\Psi^{\varepsilon, 1}(x)$.

The statements of the lemma for $\lambda_{n-2}^{(1)}, \psi_{n-2}^{(1)}(x), v_{0}^{(1)}(\xi)$ have already been proved. We recall that since the functions $\psi_{0}^{(s)}(x), v_{0}^{(1)}(\xi)$ and $\psi_{n-2}^{(1)}(x)$ are defined, the harmonic polynomials $X_{k}^{(0, s)}, Y_{k}^{(0,1)}$ and $X_{k}^{(n-2,1)}, k \geqslant 0$, are known as well.

It follows from the definition of $\Phi_{i}^{(1)}(\xi)$ that

$$
\begin{aligned}
& \Phi_{1}^{(1)}(\xi)=X_{1}^{(0,1)}(\xi)+X_{0}^{(1,1)}+\sum_{k=0}^{\infty} Y_{k}^{(1,1)}(\xi) \rho^{-2 k-1}, \quad n=3, \\
& \Phi_{1}^{(1)}(\xi)=X_{1}^{(0,1)}(\xi)+\sum_{k=0}^{\infty} Y_{k}^{(1,1)}(\xi) \rho^{-2 k-n+2}, \quad n>3,
\end{aligned}
$$

and

$$
\begin{aligned}
& \Phi_{2}^{(1)}(\xi)=X_{2}^{(0,1)}(\xi)+X_{1}^{(1,1)}+X_{0}^{(2,1)}+\sum_{k=0}^{\infty} Y_{k}^{(2,1)}(\xi) \rho^{-2 k-1}+\alpha_{1}^{(1)} X_{1}^{(0,2)}(\xi), \quad n=3, \\
& \Phi_{2}^{(1)}(\xi)=X_{2}^{(0,1)}(\xi)+X_{0}^{(1,1)}+\sum_{k=0}^{\infty} Y_{k}^{(2,1)}(\xi) \rho^{-2 k-2}+\alpha_{1}^{(1)} X_{1}^{(0,2)}(\xi), \quad n=4, \\
& \Phi_{2}^{(1)}(\xi)=X_{2}^{(0,1)}(\xi)+\sum_{k=0}^{\infty} Y_{k}^{(2,1)}(\xi) \rho^{-2 k-n+2}+\alpha_{1}^{(1)} X_{1}^{(0,2)}(\xi), \quad n>4,
\end{aligned}
$$

where $Y_{k}^{(1,1)}, Y_{k}^{(2,1)}, X_{2}^{(0,1)}$ are still arbitrary harmonic polynomials, and $\alpha_{1}^{(1)}$ is an arbitrary constant.

By Lemma 4.1 there exists a solution $v_{1}^{(1)} \in C^{\infty}\left(\mathbb{R}^{n} \backslash \bar{\omega}\right)$ to boundary value problem 6.4 having differentiable asymptotic expansion 6.20 with some harmonic polynomials $Y_{k}^{(1,1)}(\xi)$.

In particular, having determined $Y_{0}^{(1,1)}(\xi)$, by Corollary 2 we obtain that there exists a function $\psi_{n-1}^{(1)} \in \mathcal{A}$ solving problem 6.14$), 6.13$ for some $\lambda_{n-1}^{(1)}$ and $\alpha_{1}^{(1)}$ and having differentiable asymptotic expansion 6.21 with some harmonic polynomials $X_{k}^{(n-1,1)}(x)$.

In its turn, once $\alpha_{1}^{(1)}$ and $X_{2}^{(0,1)}$ are determined, we see that in 6.23 only $Y_{k}^{(2,1)}$ are still arbitrary. By Lemma 4.1 there exists a solution $v_{2}^{(1)} \in C^{\infty}\left(\mathbb{R}^{n} \backslash \bar{\omega}\right)$ to boundary value problem 6.4 having differentiable asymptotic expansion 6.20 for some harmonic polynomials $Y_{k}^{(2,1)}(\xi)$.

Once we find $Y_{0}^{(2,1)}(\xi)$ (and also $Y_{1}^{(1,1)}(\xi), Y_{2}^{(0,1)}(\xi)$ before), by Lemma 4.3 we obtain that there exists a function $\psi_{n}^{(1)} \in \mathcal{A}$ solving problem 6.14), 6.13 for some $\lambda_{n}^{(1)}$ and $\alpha_{2}^{(1)}$ and having differentiable asymptotic expansion 6.21 for some harmonic polynomials $X_{k}^{(n, 1)}(x)$ and so forth.

We proceed to constructing formal asymptotic expansions for the eigenvalue $\lambda_{\varepsilon}^{(2)}$ and the associated eigenfunction $\psi_{\varepsilon}^{(2)}(x)$. Since $\psi_{\varepsilon}^{(2)}(0)=0$, similar to (2.7), 2.9), 2.10) and (5.1), 
(5.25), (5.2), in the critical case $\psi_{0}(0)=0$, given a simple eigenvalue $\lambda_{0}$, we construct asymptotic expansion for the eigenvalue $\lambda_{\varepsilon}^{(2)}$ and the internal expansion for the eigenfunctions as (2.17) and

$$
V^{(2)}(\xi, \varepsilon)=\varepsilon v_{1}^{(2)}(\xi)+\sum_{k=2}^{\infty} \varepsilon^{k} v_{k}^{(2)}(\xi)
$$

where

$$
\begin{aligned}
& v_{1}^{(2)}(\xi) \sim X_{1}^{(0,2)}(\xi)=\sum_{m=1}^{n} \frac{\partial \psi_{0}^{(2)}}{\partial x_{m}}(0) \xi_{m}, \quad \rho \rightarrow \infty, \\
& v_{k}^{(2)}(\xi) \sim X_{k}^{(0,2)}(\xi), \quad k \geqslant 2, \quad \rho \rightarrow \infty .
\end{aligned}
$$

Substituting 6.24) into (2.2), passing to the variable $\xi$ and equating the coefficients at the like powers of $\varepsilon$, we obtain boundary value problems for $v_{k}^{(1)}$ :

$$
\Delta_{\xi} v_{k}^{(2)}=0 \quad \xi \in \mathbb{R}^{n} \backslash \bar{\omega}, \quad v_{k}^{(2)}=0 \quad \xi \in \partial \omega .
$$

Similar to the external expansion (5.8), (5.25) of the eigenfunction in the critical case $\psi_{0}(0)=$ 0 for a simple eigenvalue $\lambda_{0}$ and similar to external expansion 6.11 for the eigenfunction $\psi_{\varepsilon}^{(1)}(x)$ for a multiple eigenvalue $\lambda_{0}$, we seek the external expansion for the eigenfunction $\psi_{\varepsilon}^{(2)}(x)$ as

$$
U^{(2)}(x, \varepsilon)=\psi_{0}^{(2)}(x)+\varepsilon^{n-1} \sum_{i=0}^{\infty} \varepsilon^{i} \psi_{i+n-1}^{(2)}(x)+\varepsilon \psi_{0}^{(1)}(x) \sum_{i=0}^{\infty} \alpha_{i+1}^{(2)} \varepsilon^{i} .
$$

By matching the first term and the last sum in series (6.27) with series (6.24) we specify asymptotics 6.25 :

$$
\begin{aligned}
& v_{1}^{(2)}(\xi) \sim X_{1}^{(0,2)}(\xi)+\alpha_{1}^{(2)} X_{0}^{(0,1)}=\sum_{m=1}^{n} \frac{\partial \psi_{0}^{(2)}}{\partial x_{m}}(0) \xi_{m}+\alpha_{1}^{(2)} \psi_{0}^{(1)}(0), \quad \rho \rightarrow \infty, \\
& v_{k}^{(2)}(\xi) \sim X_{k}^{(0,2)}(\xi)+\sum_{j=1}^{k} \alpha_{j}^{(2)} X_{k-j}^{(0,1)}(\xi), \quad k \geqslant 2, \quad \rho \rightarrow \infty .
\end{aligned}
$$

It follows from the definition of the functions $z_{q}(\xi)$ that the function

$$
v_{1}^{(2)}(\xi)=\sum_{m=1}^{n} \frac{\partial \psi_{0}^{(2)}}{\partial x_{m}}(0)\left(\xi_{m}-z_{m}(\xi)\right)+\alpha_{1}^{(2)} \psi_{0}^{(1)}(0)\left(1-z_{0}(\xi)\right)
$$

is a solution to boundary value problem (6.26) and it has the differentiable asymptotic expansion

$$
v_{1}^{(2)}(\xi)=X_{1}^{(0,2)}(\xi)+\alpha_{1}^{(2)} X_{0}^{(0,1)}+\sum_{k=0}^{\infty} Y_{k}^{(1,2)}(\xi) \rho^{-2 i-n+2}, \quad \rho \rightarrow \infty
$$

where

$$
\begin{aligned}
Y_{0}^{(1,2)} & =-\sum_{m=1}^{n} \frac{\partial \psi_{0}^{(2)}}{\partial x_{m}}(0) c_{m, 0}-\alpha_{1}^{(2)} \psi_{0}^{(1)}(0) c(\omega), \\
Y_{1}^{(1,2)}(\xi) & =-\sum_{m=1}^{n} \frac{\partial \psi_{0}^{(2)}}{\partial x_{m}}(0) \sum_{q=1}^{n} c_{m, q} \xi_{q}-\alpha_{1}^{(2)} \psi_{0}^{(1)}(0) \sum_{q=1}^{n} c_{0, q} \xi_{q}, \\
Y_{k}^{(1,2)}(\xi) & =-\sum_{m=1}^{n} \frac{\partial \psi_{0}^{(2)}}{\partial x_{m}}(0) Z_{k}^{(m)}(\xi)-\alpha_{1}^{(2)} \psi_{0}^{(1)}(0) Z_{k}^{(0)}(\xi), \quad k \geqslant 2,
\end{aligned}
$$

which specifies required asymptotics 6.28 for $v_{1}^{(2)}(\xi)$. 
Rewriting the asymptotic expansion at infinity of the function $\varepsilon v_{1}^{(2)}(\xi)$ in external variables, we obtain the leading terms for the asymptotics at zero for the coefficients $\psi_{i+n-1}^{(2)}(x)$ of the external expansion:

$$
\psi_{n-1+j}^{(2)}(x) \sim Y_{j}^{(1,2)}(x) r^{-n-2 j+2}, \quad r \rightarrow 0, \quad j \geqslant 0 .
$$

Substituting series (6.27) and (2.17) into (2.2), we obtain the boundary value problem for $\psi_{n-1}^{(2)}(x)$ :

$$
\begin{aligned}
-\Delta \psi_{n-1}^{(2)} & =0, \quad x \in \Pi \backslash\{0\}, \quad \mathfrak{l} \psi_{n-1}^{(2)}=0, \quad x \in \partial \Pi \backslash \bar{\Sigma}_{a}, \\
\frac{\partial \psi_{n-1}^{(2)}}{\partial \nu} & =\lambda_{0} \psi_{n-1}^{(2)}+\lambda_{n-1}^{(2)} \psi_{0}^{(2)}, \quad x \in \Sigma_{a},
\end{aligned}
$$

where $\lambda_{n-1}^{(2)}=0$. But if $Y_{0}^{(1,2)} \neq 0$, then by Corollary 2 problem 6.35, 6.34 is insolvable for all $\lambda_{n-1}^{(2)}$. Hence, in view of 6.31), 6.32 we obtain that $Y_{0}^{(1,2)}=0$,

$$
\begin{aligned}
& \psi_{n-1}^{(2)}(x) \equiv 0, \\
& \alpha_{1}^{(2)}=-\frac{1}{\psi_{0}^{(1)}(0) c(\omega)} \sum_{m=1}^{n} \frac{\partial \psi_{0}^{(2)}}{\partial x_{m}}(0) c_{m, 0}, \\
& Y_{1}^{(1,2)}(\xi)=-\sum_{m=1}^{n} \frac{\partial \psi_{0}^{(2)}}{\partial x_{m}}(0) \sum_{q=1}^{n} c_{m, q} \xi_{q}+\frac{1}{c(\omega)} \sum_{m=1}^{n} \frac{\partial \psi_{0}^{(2)}}{\partial x_{m}}(0) c_{m, 0} \sum_{q=1}^{n} c_{0, q} \xi_{q} .
\end{aligned}
$$

We stress that once we determine $\alpha_{1}^{(2)}$, by 6.29, $6.31,6.32,6.33$ we find completely $v_{1}^{(2)}$ and $Y_{j}^{(1,2)}, j \geqslant 0$.

Substituting series (6.27) and (2.17) into (2.2), we obtain the following boundary value problems for the coefficients of external expansion (6.11):

$$
\begin{aligned}
&-\Delta \psi_{n}^{(2)}=0, \quad x \in \Pi \backslash\{0\}, \quad \mathfrak{l} \psi_{n}^{(2)}=0, \quad x \in \partial \Pi \backslash \bar{\Sigma}_{a}, \\
& \frac{\partial \psi_{n}^{(2)}}{\partial \nu}=\lambda_{0} \psi_{n}^{(2)}+\lambda_{n}^{(2)} \psi_{0}^{(2)}, \quad x \in \Sigma_{a}, \\
&-\Delta \psi_{n+i}^{(2)}=0, \quad x \in \Pi \backslash\{0\}, \quad \mathfrak{l} \psi_{n+i}^{(2)}=0, \quad x \in \partial \Pi \backslash \bar{\Sigma}_{a}, \\
& \frac{\partial \psi_{n+i}^{(2)}}{\partial \nu}=\lambda_{0} \psi_{n+i}^{(2)}+\psi_{0}^{(1)} \sum_{p=1}^{i} \alpha_{p}^{(2)} \lambda_{i+n-p}^{(2)}+\lambda_{n+i}^{(2)} \psi_{0}^{(2)}, \quad x \in \Sigma_{a}, 1 \leqslant i \leqslant n-1, \quad x \in \partial \Pi \backslash \bar{\Sigma}_{a}, \\
&-\Delta \psi_{n+i}^{(2)}\left.=0, \quad x \in \prod \backslash 0\right\}, \quad \mathfrak{l} \psi_{n+i}^{(2)}=0, \quad x \in{ }_{i}, \quad{ }^{(2)} \\
& \frac{\partial \psi_{n+i}}{\partial \nu}=\lambda_{0} \psi_{n+i}^{(2)}+\sum_{k=0}^{i-n} \lambda_{n+k}^{(2)} \psi_{i-k}^{(2)}+\psi_{0}^{(1)} \sum_{p=1}^{i} \alpha_{p}^{(2)} \lambda_{i+n-p}^{(2)}+ \\
&+\lambda_{n+i}^{(2)} \psi_{0}^{(2)}+\alpha_{i}^{(2)} \lambda_{n}^{(1)} \psi_{0}^{(1)}, \quad x \in \Sigma_{a}, i \geqslant n .
\end{aligned}
$$

By Lemma 4.1 and the definition of function $z_{0}$, one can see easily that for each number $Y_{0}^{(2,2)}$ and for some $\mu_{2}^{(2)}$ there exists a solution $v_{2}^{(2)}$ to boundary value problem 6.26 with the differentiable asymptotic expansion at infinity:

$$
\begin{aligned}
v_{2}^{(2)}(\xi)= & X_{2}^{(0,2)}(\xi)+\alpha_{1}^{(2)} X_{1}^{(0,1)}(\xi)+\alpha_{2}^{(2)} X_{0}^{(0,1)}(\xi)+ \\
& +Y_{0}^{(2,2)} \rho^{-n+2}+\sum_{j=1}^{\infty} Y_{j}^{(2,2)}(\xi) \rho^{-n+2-2 j}
\end{aligned}
$$

where $Y_{j}^{(2,2)}$ are some polynomials $j \geqslant 1$. This expansion specifies asymptotics 6.28. 
By rewriting asymptotic expansion at infinity for the function $\varepsilon^{2} v_{2}^{(2)}(\xi)$ in the external variables specifies asymptotics at zero (6.34) for the coefficients of the external expansion:

$$
\begin{aligned}
\psi_{n}^{(2)}(x) & \sim Y_{1}^{(1,2)}(x) r^{-n}+Y_{0}^{(2,2)} r^{-n+2}, \quad r \rightarrow 0, \\
\psi_{n+j}^{(2)}(x) & \sim Y_{j+1}^{(1,2)}(x) r^{-n-2 j}+Y_{j}^{(2,2)} r^{-n-2 j+2}, \quad r \rightarrow 0, \quad j \geqslant 1 .
\end{aligned}
$$

By 6.38 and Corollary 3 the function

$$
\psi_{n}^{(2)}(x)=-\sum_{m=1}^{n} \frac{\partial \psi_{0}^{(2)}}{\partial x_{m}}(0) \sum_{q=1}^{n} c_{m, q} \widetilde{E}_{q}(x)+\frac{1}{c(\omega)} \sum_{m=1}^{n} \frac{\partial \psi_{0}^{(2)}}{\partial x_{m}}(0) c_{m, 0} \sum_{q=1}^{n} c_{0, q} \widetilde{E}_{q}(x) \in \mathcal{A}
$$

is a solution to boundary value problem 6.39 for $\lambda_{n}^{(2)}$ determined by identity 2.19 and has the differentiable asymptotic expansion

$$
\psi_{n}^{(2)}(x)=Y_{1}^{(1,2)}(x) r^{-n}+Y_{0}^{(2,2)} r^{-n+2}+\sum_{j=0}^{\infty} X_{j}^{(n, 2)}(x), \quad r \rightarrow 0,
$$

for some explicitly calculated $Y_{0}^{(2,2)}$. This expansion specifies asymptotics 6.43). We stress that by determining $Y_{0}^{(2,2)}$ we also find $\mu_{2}^{(2)}$ and, therefore, we have completely found $v_{2}^{(2)}(\xi)$.

At the next step of matching by the solvability condition of problem $6.40,66.41$ for $\psi_{n+1}^{(2)}$ by Lemma 4.4 we find $\mu_{3}^{(2)}, \lambda_{n+1}^{(2)}, \psi_{n+1}^{(2)}, v_{3}^{(2)}$ and specify the asymptotics at zero for the coefficients of external expansion $\psi_{n+1+j}^{(2)}$ as $j \geqslant 1$ and so forth.

As a result, we arrive at the following statement.

Lemma 6.2. There exist series (2.17), (6.27), (6.36), (6.24) such that the coefficients of series (6.27) belong to $\mathcal{A}$, solve boundary value problems (6.39), (6.40), (6.41) and have differentiable asymptotic expansions at zero:

$$
\begin{aligned}
\psi_{0}^{(1)}(x) & =\sum_{k=0}^{\infty} X_{k}^{(0,1)}(x), \quad \psi_{0}^{(2)}(x)=\sum_{k=1}^{\infty} X_{k}^{(0,2)}(x), \\
\psi_{n+i}^{(2)}(x) & =\sum_{k=0}^{i+1} Y_{k}^{(i+2-k .2)}(x) r^{-2 k-n+2}+\sum_{k=0}^{\infty} X_{k}^{(n+i, 2)}(x), \quad i \geqslant 0,
\end{aligned}
$$

while the coefficients of series (6.24) belong to $C^{\infty}\left(\mathbb{R}^{n} \backslash \bar{\omega}\right)$, are solutions to boundary value problems (6.26) and at infinity, they have the differentiable asymptotic expansions:

$$
\begin{aligned}
& v_{i}^{(2)}(\xi)=X_{i}^{(0,2)}(\xi)+\sum_{k=0}^{\infty} Y_{k}^{(i, 2)}(\xi) \rho^{-2 k-n+2}+\sum_{k=0}^{i-1} \alpha_{k+1}^{(2)} X_{i-k-1}^{(0,1)}(\xi), \quad 1 \leqslant i<n, \\
& v_{i}^{(2)}(\xi)=X_{i}^{(0,2)}(\xi)+\sum_{k=0}^{i-n} X_{k}^{(i-k, 2)}(\xi)+\sum_{k=0}^{\infty} Y_{k}^{(i, 2)}(\xi) \rho^{-2 k-n+2}+\sum_{k=0}^{i-1} \alpha_{k+1}^{(2)} X_{i-k-1}^{(0,1)}(\xi), \quad i \geqslant n .
\end{aligned}
$$

Identities (2.19), 6.44), 6.29), 6.37) hold true.

We denote by $\hat{\lambda}_{\varepsilon, N}^{(1)}, \widehat{u}_{\varepsilon, N}^{(1)}(x), \widehat{v}_{\varepsilon, N}^{(1)}(\xi)$ partial sums of series $(2.16, \sqrt{6.11}$ and 6.2$)$ up to powers $N$ and by $\widehat{\lambda}_{\varepsilon, N}^{(2)}, \widehat{u}_{\varepsilon, N}^{(2)}(x), \widehat{v}_{\varepsilon, N}^{(2)}(\xi)$ we denote partial sums of series (2.17), 6.27), 6.36), 6.24). Lemmata 6.1, 6.2 imply the following statement.

Lemma 6.3. The function $\widehat{u}_{\varepsilon, N}^{(i)} \in \mathcal{A}$ solves the problem

$$
\begin{aligned}
-\Delta \widehat{u}_{\varepsilon, N}^{(i)}(x) & =0, \quad x \in \Pi \backslash\{0\}, \quad \mathfrak{l} \widehat{u}_{\varepsilon, N}^{(i)}(x)=0, \quad x \in \partial \Pi \backslash \bar{\Sigma}_{a}, \\
\frac{\partial \widehat{u}_{\varepsilon, N}^{(i)}(x)}{\partial \nu} & =\widehat{\lambda}_{\varepsilon, N}^{(i)} \widehat{u}_{\varepsilon, N}^{(i)}(x)+O\left(\varepsilon^{N+1}\right), \quad x \in \Sigma_{a} .
\end{aligned}
$$


The convergence

$$
\int_{\Sigma_{a}}\left(\widehat{u}_{\varepsilon, N}^{(i)}-\psi_{0}^{(i)}\right)^{2} d x^{\prime} \rightarrow 0, \quad \varepsilon \rightarrow 0
$$

holds true.

The function $\widehat{v}_{\varepsilon, N}^{(i)} \in C^{\infty}\left(\mathbb{R}^{n} \backslash \omega\right)$ is a solution to the boundary value problem

$$
\Delta_{\xi} \widehat{v}_{\varepsilon, N}^{(i)}(\xi)=0, \quad \xi \in \mathbb{R}^{n} \backslash \bar{\omega}, \quad \widehat{v}_{\varepsilon, N}^{(i)}(\xi)=0, \quad \xi \in \partial \omega .
$$

As $\varepsilon^{\frac{1}{2}}<r<2 \varepsilon^{\frac{1}{2}}$ (or, the same, as $\varepsilon^{-\frac{1}{2}}<\rho<2 \varepsilon^{-\frac{1}{2}}$ ) the differentiable identity

$$
\widehat{u}_{\varepsilon, N}^{(i)}(x)-\widehat{v}_{\varepsilon, N}^{(i)}(\xi)=O\left(r^{N+1}+\varepsilon^{N} r+\rho^{-N-1}+\varepsilon^{N} \rho^{-1}\right)
$$

holds true.

We denote

$$
\widetilde{u}_{\varepsilon, N}^{(i)}(x)=\tilde{\chi}\left(r \varepsilon^{-\frac{1}{2}}\right) \widehat{u}_{\varepsilon, N}^{(i)}(x)+\left(1-\tilde{\chi}\left(r \varepsilon^{-\frac{1}{2}}\right)\right) \widehat{v}_{\varepsilon, N}^{(i)}\left(\frac{x}{\varepsilon}\right) .
$$

Similar to Lemma 5.3 but using Lemma 6.3 instead of Lemma 5.2 one can prove the following statement.

Lemma 6.4. The function $\widetilde{u}_{\varepsilon, N}^{(i)} \in H^{1}\left(\Pi_{\varepsilon}\right)$ solves boundary value problem

$$
\begin{aligned}
& -\Delta \widetilde{u}_{\varepsilon, N}^{(i)}=F_{\varepsilon, N}^{(i)}, \quad x \in \Pi_{\varepsilon}, \quad \mathfrak{u}_{\varepsilon, N}^{(i)}=0, \quad x \in \partial \Pi \backslash \bar{\Sigma}_{a}, \\
& \frac{\partial \widetilde{u}_{\varepsilon, N}^{(i)}}{\partial \nu}=\widehat{\lambda}_{\varepsilon, N}^{(i)} \widetilde{u}_{\varepsilon, N}^{(i)}+g_{\varepsilon, N}^{(i)}, \quad x \in \Sigma_{a}, \quad \widetilde{u}_{\varepsilon, N}^{(i)}=0, \quad x \in \partial \omega_{\varepsilon},
\end{aligned}
$$

where

$$
\begin{aligned}
& \left\|g_{\varepsilon, N}^{(i)}\right\|_{L_{2}\left(\Sigma_{a}\right)} \leqslant C \varepsilon^{N+1}, \\
& \left\|F_{\varepsilon, N}^{(i)}\right\|_{L_{2}\left(\Pi_{\varepsilon}\right)} \leqslant C \varepsilon^{\frac{2 N+n-2}{4}}, \\
& \operatorname{supp} F_{\varepsilon, N}^{(i)} \subset \bar{B}_{2 \varepsilon^{\frac{1}{2}}} \backslash B_{\varepsilon^{\frac{1}{2}}} .
\end{aligned}
$$

Multiplying equation 6.48 by the eigenfunction $\psi_{\varepsilon}^{(j)}$ and integrating by parts over $\Pi_{\varepsilon}$, by boundary value problems (6.48), (2.2) we obtain

$$
\left(\lambda_{\varepsilon}^{(j)}-\widehat{\lambda}_{\varepsilon, N}^{(i)}\right) \int_{\Sigma_{a}} \widetilde{u}_{\varepsilon, N}^{(i)} \psi_{\varepsilon}^{(j)} d x^{\prime}=\int_{\Pi_{\varepsilon}} F_{\varepsilon, N}^{(i)} \psi_{\varepsilon}^{(j)} d x+\int_{\Sigma_{a}} g_{\varepsilon, N}^{(i)} \psi_{\varepsilon}^{(j)} d x^{\prime} .
$$

Similar to $(5.49)$ and (5.50) we obtain that

$$
\int_{\Sigma_{a}} g_{\varepsilon, N}^{(i)} \psi_{\varepsilon}^{(j)} d x^{\prime}=O\left(\varepsilon^{N+1}\right), \quad \int_{\Pi_{\varepsilon}} F_{\varepsilon, N}^{(i)} \psi_{\varepsilon}^{(j)} d x=O\left(\varepsilon^{\frac{2 N+n-2}{4}}\right) .
$$

It follows from Theorem 2.1 that in each sequence $\varepsilon_{k} \rightarrow 0$ we can choose a subsequence $\varepsilon_{k_{m}}$ such that on this subsequence, the convergences

$$
\psi_{\varepsilon}^{(i)} \rightarrow \alpha_{1}^{(i)} \psi_{0}^{(1)}+\alpha_{2}^{(i)} \psi_{0}^{(2)}, \quad\left(\alpha_{1}^{(i)}\right)^{2}+\left(\alpha_{2}^{(i)}\right)^{2}=1, \quad \alpha_{1}^{(1)} \alpha_{1}^{(2)}+\alpha_{2}^{(1)} \alpha_{2}^{(2)}=0
$$

hold. Assume that $\alpha_{1}^{(1)} \alpha_{2}^{(1)} \neq 0$. Then $\alpha_{1}^{(2)} \alpha_{2}^{(2)} \neq 0$ and it follows from 6.52, 6.53), 6.46) that

$$
\lambda_{\varepsilon}^{(1)}-\widehat{\lambda}_{\varepsilon, N}^{(1)}=O\left(\varepsilon^{\frac{2 N+n-2}{4}}\right), \quad \lambda_{\varepsilon}^{(1)}-\widehat{\lambda}_{\varepsilon, N}^{(2)}=O\left(\varepsilon^{\frac{2 N+n-2}{4}}\right), \quad \forall N
$$


The above is impossible since $\left|\widehat{\lambda}_{\varepsilon, N}^{(1)}-\widehat{\lambda}_{\varepsilon, N}^{(2)}\right| \geqslant c \varepsilon^{2}$, where $c>0$, by 2.16), 2.17) and 2.18). Therefore, $\alpha_{1}^{(1)} \alpha_{2}^{(1)}=\alpha_{1}^{(2)} \alpha_{2}^{(2)}=0$. Since the original sequence $\varepsilon_{k}$ is arbitrary, it follows that

$$
\left\|\psi_{\varepsilon}^{(i)}-\psi_{0}^{(i)}\right\|_{L_{2}\left(\Sigma_{a}\right)} \rightarrow 0, \quad \varepsilon \rightarrow 0
$$

And finally, (6.52) as $j=i,(6.53)$ and 6.54$)$ yield that

$$
\lambda_{\varepsilon}^{(i)}-\widehat{\lambda}_{\varepsilon, N}^{(i)}=O\left(\varepsilon^{\frac{2 N+n-2}{4}}\right) .
$$

Since $N$ is arbitrary, it implies expansions (2.16), (2.17). The proof of Theorem 2.3 is complete.

\section{BIBLIOGRAPHY}

1. A.A. Samarskii. "On the influence of constraints on the characteristic frequencies of closed volumes" // Dokl. Akad. Nauk SSSR. 63:6, 631-634 (1948) (in Russian).

2. Yu.N. Dnestrovsky. On varying of eigenvalues under variation of the boundaty of a domain // Vestnik Mosk. Univ. Ser. I. Matem. Mekh. 9, 61-74 (1964) (in Russian).

3. Sh. Ozawa. Singular Hadamard's variation of domains and eigenvalues of Laplacian // Proc. Jap. Acad. Ser. A. 56:7, 351-357 (1980).

4. C.A. Swanson. Asymptotic variontional formulae for eigenvalues // Canad. Math. Bull. 6:1, 15-25 (1963).

5. V.G. Maz'ya, S.A. Nazarov, and B.A. Plamenevskii. Asymptotic expansions of the eigenvalues of boundary-value problems for the Laplace operator in domains with small holes // Izv. Akad. Nauk SSSR. Ser. Mat. 48:2, 347-371 (1984). [Math. USSR-Izv. 24:2, 321-345 (1985)].

6. A.M. Il'in. An investigation of the asymptotics for a solution of an elliptic boundary value problem in a domain with a small hole // Trudy Semin. I.G. Petrovskogo. 6, 57-82 (1981). (in Russian).

7. I. V. Kamotskii and S. A. Nazarov. Spectral problems in singularly perturbed domains and selfadjoint extensions of differential operators // Tr. St.-Peterbg. Mat. Obshch. 6. 151-212 (1998) (in Russian).

8. D.B. Davletov. Singularly perturbed Dirichlet boundary-value problem for a stationary system in the linear elasticity theory // Izv. Vyssh. Uchebn. Zaved. Mat., 12, 7-16 (2008) [Russian Math. Izv. VUZ. 52:12, 4-12 (2008)].

9. D.B. Davletov. Asymptotics of eigenvalues of the Dirichlet boundary-value problem for the Lame operator in a three-dimensional domain with a small cavity // Zhurn. Vych. Matem. Matem. Fiz. 48:10, 1847-1858 (2008) [Comput. Math. and Math. Phys. 48:10, 1811-1822 (2008)].

10. D.B. Davletov. Asymptotics of eigenvalues of the two-dimensional Dirichlet boundary-value problem for the Lamé operator in a domain with a small hole // Matem. Zametki. 93:4, 537-548 (2013).

11. S.A. Nazarov. Asymptotic expansions of eigenvalues of the Steklov problem in singularly perturbed domains // Alg. Anal. 26:2, 119-184 (2014). [St. Petersburg Math. J. 26:2, 273-318 (2015).]

12. R.R. Gadyl'shin, D.V. Kozhevnikov. Homogenization of the boundary value problem in a domain perforated along a part of the boundary // Probl. Mat. Anal. 75, 41-59 (2014). [J. Math. Sci. 198:6, 701-723 (2014).]

13. G.A. Chechkin. Averaging of boundary value problems with a singular perturbation of the boundary conditions // Matem. Sborn. 84:6, 99-150 (1993). [Sb. Math. 79:1, 191-222 (1994).]

14. R.R. Gadyl'shin, G.A. Chechkin. A boundary value problem for the Laplacian with rapidly changing type of boundary conditions in a multi-dimensional domain // Sibir. Matem. Zhurn. 40:2, 271-287. [Siber. Math. J. 40:2, 229-244 (1999).]

15. A.G. Belyaev, G.A. Chechkin, R.R. Gadyl'shin Effective membrane permeability: estimates and low concentration asymptotics // SIAM J. Appl. Math. 60:1, 84-108 (2000).

16. S.A. Nazarov. Variational and asymptotic methods for finding eigenvalues below the continuous spectrum threshold // Sibir. Matem. Zhurn. 51:5, 1086-1101 (2010). [Siber. Math. J. 51:5, 866-878 (2010).]

17. D. Borisov. On a $\mathcal{P} \mathcal{T}$-symmetric waveguide with a pair of small holes // Trudy Inst. Matem. Mekh. UrO RAN. 18:2, 22-37 (2012). [Proc. Steklov Inst. Math. 281:1, 5-21 (2013).] 
18. A.M. Il'in. Matching of asymptotic expansions of solutions of boundary value problems. Nauka, Moscow (1989). [Transl. Math. Monographs. AMS, Providence, RI (1992).]

19. R.R. Gadyl'shin. Concordance method of asymptotic expansions in a singularly-perturbed boundary-value problem for the Laplace operator // Sovrem. Mat. Prilozh. Asimptot. Metody Funkts. Anal. 3-32 (2003). [J. Math. Sci. 125:5, 579-609 (2005).]

20. A.R. Bikmetov, R.R. Gadyl'shin. Perturbation of an elliptic operator by a narrow potential in an n-dimensional domain // Ufimskij Matem. Zhurn. 4:2, 28-64 (2012). [Ufa Math. J. 4:2, 28-64 (2012).]

21. G. Pólya, G. Szegö. Isoperimetric inequalities in mathematical physics. Ann. Math. Stud. 27. Princeton Univ. Press, Princeton (1951).

22. V.P. Mikhajlov. Partial differential equations. Nauka, Moscow (1976). [Mir Publishers, Moscow (1978).]

23. T. Kato. Perturbation theory for linear operators. Springer-Verlag, Berlin (1966).

Davletov Dmitrii Borisovich,

Bashkir State Pedagogical Univeristy named after M. Akhmulla,

October rev. st. 3a,

450000, Ufa, Russia

E-mail: davletovdb@mail.ru

Kozhevnikov Denis Vladimirovich,

Bashkir State Pedagogical Univeristy named after M. Akhmulla,

October rev. st. 3a,

450000, Ufa, Russia

E-mail: kozhevnikovbspu@gmail.com 